\title{
New catalogue of Wolf-Rayet galaxies and high-excitation extra-galactic HII regions ${ }^{\star}$
}

\author{
D. Schaerer ${ }^{1}$, T. Contini ${ }^{2,3}$, and M. Pindao ${ }^{4}$ \\ 1 Observatoire Midi-Pyrénées, Laboratoire d'Astrophysique, 14 Av. E. Belin, F-31400 Toulouse, France \\ 2 School of Physics \& Astronomy, Tel Aviv University, 69978 Tel Aviv, Israel \\ 3 European Southern Observatory, Karl-Schwarzschild-Str. 2, D-85748 Garching bei München, Germany \\ ${ }^{4}$ Observatoire de Genève, 51 Ch. des Maillettes, CH-1290 Sauverny, Switzerland
}

Received October 27; accepted November 24, 1998

\begin{abstract}
We present a new compilation of Wolf-Rayet (WR) galaxies and extra-galactic HII regions showing broad HeII $\lambda 4686$ emission drawn from the literature. Relevant information on the presence of other broad emission lines (N III $\lambda 4640, \mathrm{C}$ IV $\lambda 5808$ and others) from WR stars of WN and WC subtypes, and other existing broad nebular lines is provided.

In total we include 139 known WR galaxies. Among these, 57 objects show both broad He II $\lambda 4686$ and C IV $\lambda 5808$ features. In addition to the broad (stellar) He II $\lambda 4686$ emission, a nebular He II component is well established (suspected) in 44 (54) objects. We find 19 extra-galatic HII regions without WR detections showing nebular He II $\lambda 4686$ emission.

The present sample can be used for a variety of studies on massive stars, interactions of massive stars with the ISM, stellar populations, starburst galaxies etc. The data is accessible electronically and will be updated periodically.
\end{abstract}

Key words: galaxies: starburst - galaxies: stellar content - galaxies: ISM — stars: Wolf-Rayet

\section{Introduction}

Wolf-Rayet (WR) galaxies are extragalactic objects whose integrated spectra show direct signatures from WR stars, most commonly a broad He II $\lambda 4686$ feature originating in the stellar winds of these stars. Since the first detection of such a feature in He 2-10 (Allen et al. 1976), a large

Send offprint requests to: D. Schaerer, schaerer@obs-mip.fr

* The catalogue is available at the CDS via anonymous ftp to cdsarc.u-strasbg.fr (130.79.128.5) or via http://cdsweb.ustrasbg.fr/Abstract.html number of WR galaxies have been reported, some in systematic searches (e.g. Kunth \& Joubert 1985), but mostly serendipitously. For example many objects with WR features have been found in samples of high $\mathrm{S} / \mathrm{N}$ spectra of low metallicity extra-galactic HiI regions aimed at deriving the primordial He abundance (cf. Izotov \& Thuan 1998). It must be reminded to use the term WR "galaxy" with caution. Depending e.g. on the distance of the object and the spatial extension of the observation, the region of concern may be "just" a single extra-galactic HII region with a few WR stars in a galaxy or the nucleus of a powerful starburst galaxy harbouring numerous massive stars.

Since the compilation of Conti (1991) listing 37 objects, the number of known WR galaxies has grown rapidly to more than 130 in the present catalogue. Interestingly many objects are now found showing additional features from WR stars in their spectra. E.g. the broad emission lines of N III $\lambda 4640$ and/or C III $\lambda 4650$ as well as $\mathrm{CIV} \lambda 5808$, among the strongest optical lines in WN and WC stars, are increasingly often being detected. Lines originating from WC stars (representing more evolved phases than WN stars) provide useful independent and complementary information on the massive star content in these regions (e.g. Schaerer et al. 1999).

By definition it is not surprising that WR galaxies do not form a homogeneous class. Indeed, WR galaxies are found among a large variety of morphological types, from low-mass blue compact dwarf (BCDs) and irregular galaxies, to massive spirals and luminous merging IRAS galaxies. Recent studies also quite convincingly show the evidence of signatures from WR stars in Seyfert 2 and LINERs (Osterbrock \& Cohen 1982; Ho et al. 1995; Heckman et al. 1997; Storchi-Bergmann et al. 1998; Kunth \& Contini 1998). Allen (1995) claims even the possible detection of WR stars in central cluster galaxies of two cooling flows out to a redshift of $z \sim 0.25$. 
Empirically all WR galaxies show nebular emission lines. The absolute scales (absolute magnitudes, ionizing fluxes etc.) of the investigated objects vary greatly; generally speaking the properties of WR galaxies overlap with those of other emission line galaxies and form a continuous extension of giant Hir regions (Conti 1991).

For most "traditional" WR galaxies (e.g. Hiı galaxies, BCDs etc.) the nebular spectrum is likely due to photoionization of stellar origin. However, this statement does obviously not hold in general, e.g. for Seyfert 2 and LINER revealing the presence of WR stars. Among the former "class" a considerable fraction $(\sim 1 / 3$ in the present compilation) of objects also show nebular He II $\lambda 4686$ emission in addition to the broad WR feature. This line is also present in some giant HII regions where no WR features have been detected. Except in planetary nebulae, nebular He II $\lambda 4686$ emission is very rarely found in Galactic HII regions (cf. Garnett et al. 1991; Schaerer 1997). Its origin, requiring sources with sufficient photons of energy $>54 \mathrm{eV}$, has remained puzzling (see Garnett et al. 1991 and references therein). Supported by quantitative modeling, Schaerer (1996) has suggested that the origin of nebular He II $\lambda 4686$ emission is intimately linked with the appearance of hot WR stars. To facilitate systematic analysis on the origin of nebular He II $\lambda 4686$ emission we therefore also include the relevant information on objects showing this line. Such studies have a bearing on our understanding of physical processes in HiI regions and related nebulae, the ionizing fluxes of starbursts and their contribution to the ionization of the intergalactic medium etc. (cf. Garnett et al. 1991; Schaerer et al. 1998; Stasińska 1998).

The minimum common property of all WR galaxies is (provided the origin of the considered line and our understanding of stellar evolution is correct) ongoing or recent star formation which has produced stars massive enough to evolve to the WR stage. This indicates typically ages of $\lesssim 10 \mathrm{Myr}$ and stars with initial masses $M_{\text {ini }} \gtrsim 20 M_{\odot}$ (Maeder \& Conti 1994).

WR galaxies are therefore ideal objects to study the early phases of starbursts, determine burst properties (age, duration, SFR), and to constrain parameters (i.e. slope and upper mass cut-off) of the upper part of the initial mass function (see e.g. Arnault et al. 1989; Mas-Hesse \& Kunth 1991, 1998; Krüger et al. 1992; Vacca \& Conti 1992; Meynet 1995; Contini et al. 1995; Schaerer 1996; Schaerer et al. 1999). Conversely studies of the stellar populations in super star clusters frequently formed in starbursts and WR galaxies (Conti \& Vacca 1994; Meurer et al. 1995) can also place constraints on stellar evolution models for massive stars, e.g. at extremely low metallicities, which are inaccessible in the Local Group (cf. I Zw 18: Izotov et al. 1997b; Legrand et al. 1997; de Mello et al. 1998).

As galaxies exhibiting intense star formation are being discovered in large numbers at progressively larger distances, "template" systems become increasingly impor- tant for our understanding of distant objects which cannot be studied to the same depth. As such WR galaxies represent useful templates of young starbursts which show close resemblance to recently discovered high redshift galaxies (cf. Leitherer et al. 1996; Ebbels et al. 1996; Lowenthal et al. 1997).

The present compilation should facilitate future systematic studies on some of the issues discussed above. The structure of the paper is as follows. In Sect. 2 we review the searches undertaken up to date for WR galaxies. In Sect. 3 the compilation of all known WR galaxies is presented. Brief remarks on each individual object are given in Sect. 4. The list of extra-galactic HiI regions showing only nebular He II $\lambda 4686$ is given in Sect. 5 . Suspected WR galaxies are discussed in Sect. 6 . A brief discussion and our main conclusions are found in Sect. 7.

\section{Searches for WR galaxies}

Few systematic searches for WR populations outside the Local Group (or "WR galaxies") have been undertaken. In this section we briefly summarise the studies explicitly devoted to the detection of WR signatures. A list of candidate WR galaxies resulting from some of these searches or found loosely in the literature is provided in Sect. 6. The WR galaxies issued from the searches described below are included in our list and represent the vast majority of detections. Let us now briefly summarise the properties of the spectroscopic and narrow-band imaging searches.

\subsection{Spectroscopic searches}

The first search for WR features in giant HII regions of nearby galaxies was carried out by D'Odorico et al. (1983). The latest update from their study is summarised by Rosa \& D'Odorico (1986). Data from their work was included in the quantitative analysis by Arnault et al. (1989).

The most detailed search was undertaken by Kunth \& Joubert (1985) from a sample of 45 "lazy" galaxies (blue emission-line galaxies forming stars by intermittent short bursts) from various sources. In their statistical approach they measure the excess emission above the continuum between $4600-4711 \AA$ (rest wavelength) after subtracting a typical nebular contamination taken as a function of the excitation level and abundance. Their search yielded 19 regions ( 15 different objects) with excess emission above $0.8 \sigma$.

A systematic search for a broad WR bump in all the HII galaxies included in the catalogue of Terlevich et al. (1991) was presented by Masegosa et al. (1991). Earlier publications using a subset of the same observational data had also reported some WR detections and nebular He II $\lambda 4686$ (Campbell \& Smith 1986; Campbell et al. 1986). Positive detections were considered by Masegosa et al. 
when the "blue bump" was at least $1 \sigma$ over the continuum level and clearly discernible from the nebular He II $\lambda 4686$ line. Their search yielded 37 detections $(\sim 10 \%$ of the sample); 14 of these objects have spectra with a spectral resolution of $\lesssim 5 \AA$ FWHM, which the authors estimate to be "good enough" to reliably detect WR stars. Only these objects (their Table 2) were included in our list as confirmed WR galaxies. The remaining objects are classified here as "candidates" (Sect. 6).

Recently Pindao (1998) and Pindao et al. (1999) have reanalysed the spectra from the Terlevich et al. catalogue and $\gtrsim 100$ additional emission line galaxies for their WR content. Objects with a clear detection of broad He II $\lambda 4686$ are retained as WR galaxies here (See Pindao 1998. The detection level corresponds to $\gtrsim 0.8 \sigma$, i.e. category 4 of Pindao et al. 1999). Category 3 objects (WR bump detection at $\sim 0.5 \sigma$ ) from Pindao et al. (1999) are classified here as "WR candidates".

Robledo-Rella \& Conti (1993) presented a search for WR features in a selected sample of northern HiI galaxies; candidates are given in Sect. 6 .

First results from a new search for WR signatures in young starbursts have been presented by Contini (1996) and Kovo \& Contini (1998).

An ongoing systematic search for WR galaxies has been mentioned by Huang et al. (1998).

According to Izotov (1998, private communication) the observational data gathered primarily for accurate determinations of the helium abundance since 1993 (see Izotov et al. 1994, 1996, 1997a; Thuan et al. 1995; Izotov \& Thuan 1998) are being systematically re-analysed for their WR content (Guseva et al. 1998; Izotov et al. 1998). Adding 10 newly observed objects, their sample mostly including blue compact galaxies consists of $\sim 70$ spectra. While the initial sample contained essentially very metal-poor objects, metallicities up to $\sim$ solar are now also included. The majority of the WR detections have been mentioned in the above papers; in total Guseva et al. (1998) and Izotov et al. (1998) find 41 WR galaxies, defined by broad emission between $\sim 4620-4700 \AA$. Often several broad features are pointed out in the blue bump (He II $\lambda 4686$, $\mathrm{N}$ III $\lambda 4640$, but also other lines they identify as N III $\lambda 4510, \quad \mathrm{~N}$ II $\lambda 4565, \mathrm{NV} \lambda 4605,4620, \mathrm{C}$ IV $\lambda 4658)$. According to their study 28 spectra also show broad C IV $\lambda 5808$. Finally, few detections of broad He II $\lambda 5412$, C III $\lambda 5696$, and also C II $\lambda 4267$ are signaled. We retain all except one WR galaxy (Mrk $1026=$ NGC 848 showing no broad He II $\lambda 4686$ ) from their study.

\subsection{Narrow-band imaging and others}

Drissen et al. (1993) have conducted a search for He II $\lambda 4686$ emission via narrow-band imagery in four low mass galaxies (GR8, NGC 2366, IC 2574, NGC 1569). Two of them are now confirmed WR galaxies (see above), IC 2574 remains to be studied spectroscopically, and GR8 yielded negative results (no He II).

Schmidt-Kaler \& Feitzinger (1984) initiated a search for 30 Dor and NGC 604 like objects based on POSS, ESO-Blue, and SRC film. To the best of our knowledge results from this survey have not been published.

\section{New catalogue of WR galaxies}

In this section we present an updated list of all "WR galaxies" outside the Local Group. As pointed out earlier we remind the reader that the working definition for WR galaxies refers only to the detection of a broad WR feature in an integrated spectrum, irrespectively of the included area. This fact must be taken into account in subsequent interpretations. By this working definition we also include some nearby objects (e.g. NGC 300) which, although already known to harbour WR stars, were not included in the catalogue of Conti (1991). An inventory of the Galactic WR stars is found in the 7th catalogue of van der Hucht et al. (1998; cf. van der Hucht 1996). For individual WR stars and WR populations in the Local Group see e.g. the review of Massey (1996).

\subsection{Selection criteria and procedure}

During the last three years the literature was followed for any publication relating to possible or confirmed signatures from WR stars in extragalactic objects. At the end of july 1998 we also made a systematic search in the Astrophysics Data System (ADS) for the occurrence of "WR", "W-R", "Wolf-Rayet", or "Wolf Rayet" in all the included literature starting with 1970. The search yielded 1780 references to refereed papers, and 1061 other references, all of which were carefully inspected. In addition all publications from the IAU Symposia 67, 80, 83, 88, $99,105,116,122,143,149,163$ were scanned. Although a complete inspection is not possible the present procedure should guarantee a fairly high degree of completeness.

For the inclusion in our list of "WR galaxies" the criteria are 1) the presence of broad emission at $4686 \AA$ due to He II, or 2) a broad "WR bump" at 4660 , or 3) other broad emission lines attributed to WR stars. The second criterion accounts for the difficulty of resolving He II $\lambda 4686$ from other emission lines in medium resolution spectra (see e.g. Kunth \& Joubert 1985), but may in some cases introduce objects where nebular emission lines dominate the WR bump. Implicitly both criteria 1) and 2) were adopted in the earlier compilation of Conti (1991). The third criterion allows for objects where broad carbon lines (e.g. C III $\lambda 5696$, C IV $\lambda 5808$ ) presumably from WR stars are detected. In practice only one case (NGC 1365 for which no blue spectrum was available) falls in this category. 
The presence/absence of features and their qualification (broad and/or nebular) is generally not fully objective (see Sect. 2 for more details). Furthermore in many cases the spectra are not available for inspection. We therefore follow the procedure or judgement of the authors of the original publication.

All objects resulting from this selection are listed in Table 1. Obviously most of the objects appear in various catalogues. We have chose the following labeling priority: first the Messier number, then the NGC, the Markarian, the Zwicky lists, or finally other labels (UM, Tol, SBS, etc.). Often several regions in the same object show WR features. In this case only the main object name is listed (Col. 1); information about the different regions are given in Sect. 4.

The general properties of objects, equatorial coordinates (equinox 1950, Cols. 2 and 3), morphological type (Col. 4), apparent blue magnitude (Col. 5), and heliocentric radial velocity (Col. 6) have been extracted from NED. Column 7 gives the reference of the first publication indicating the presence of broad He II $\lambda 4686$. "(C91)" indicates that this objects was already included in the compilation of Conti (1991). Column 8 gives information about the existence of nebular He II $\lambda 4686$ (see footnote of the Table for the keys). The source of this information is found under the remarks on each individual object (Sect. 4). Column 9 gives the reference to the first detection of broad C IV $\lambda 5808$ (exceptionally also other broad features, cf. NGC 1365).

Our current compilation (Table 1) includes 139 WR galaxies (cf. $37 \mathrm{WR}$ galaxies in Conti 1991). Among them 57 objects show also broad C IV $\lambda 5808$ features. In addition to the broad (stellar) He II $\lambda 4686$ emission, a nebular He II component is well established (suspected) in 44 (54) objects.

The database will be made available electronically at the CDS and through the $\mathrm{Web}^{1}$. It is our intention to update the catalogue in the future by this means.

\section{Remarks on individual WR galaxies}

Below a brief comment is given for each object from Table 1 on the detected WR features and possible nebular He II $\lambda 4686$. In some objects the strongest nebular lines also show broad and/or asymmetric components, attributed to gaz-flows or broad stellar emission. These finding are also reported below.

NGC 53 - Detection of He II $\lambda 4686$ by Masegosa et al. (1991) from automatic search in HII galaxy catalogue of Terlevich et al. (1991).

NGC 55 - Rosa \& D'odorico (1986) surveyed eight giant HII regions in NGC 55. Two were found with broad He II $\lambda 4686$ and C IV $\lambda 5808$ features.

\footnotetext{
1 information at

http://www .obs-mip.fr/omp/astro/people/schaerer
}

UM 48 - Automatic detection by Masegosa et al. (1991). WR features unlikely according to the analysis of Pindao et al. (1999).

Mrk 960 - Broad features around $4700 \AA$ were first suspected by S. Considère (private communication). A better spectrum of this galaxy confirms the presence of a broad He II $\lambda 4686$ emission line (Kovo \& Contini 1998).

NGC 300 - Fifteen positions were observed by D'Odorico et al. (1983) in this Sculptor group galaxy often considered as a twin of M 33. Two regions showed He II $\lambda 4686$ one also C IV $\lambda 5808$. Several investigations were undertaken subsequently to search for individual WR stars or small clusters. The latest work (Breysacher et al. 1997, cf. references therein) detected $12 \mathrm{WR}$ stars increasing the total to 34 known WR stars.

IRAS 01003 - 2238 - Broad He II $\lambda 4686$ and N III $\lambda 4640$ emission has been observed by Armus et al. (1988). Other broad features are tentatively detected. Based on the equivalent withs of the WR-bump they estimate $\sim 10^{5}$ WR stars are present in this luminous infrared galaxy, the most distant WR galaxy known so far.

UM 311 - This HII region is possibly located in the galaxy NGC 450. Automatic detection of broad He II $\lambda 4686$ by Masegosa et al. (1991). The high $\mathrm{S} / \mathrm{N}$ spectrum of Izotov \& Thuan (1998) shows both broad He II $\lambda 4686$ and C IV $\lambda 5808$ features, but no nebular He II. A nebular component is also detected by Guseva et al. (1998). Pindao (1998) confirms the presence of a broad WR bump.

Tol 0121 - 376 - Masegosa et al. (1991) and Pindao (1998) signal the presence of a broad WR bump in this galaxy.

Minkowski's Object — A weak broad He II $\lambda 4686$ feature and possible nebular He II has been found by Breugel et al. (1985) in this "starburst triggered by a radio jet". They also point out a close similarity of the emission line spectrum with the NGC 7714, a prototypical starburst galaxy.

Mrk 996 - This unusual blue compact galaxy shows He II $\lambda 4686$, N III $\lambda 4640$ and C IV $\lambda 5808$ features in the HST FOS spectra of Thuan et al. (1996).

Mrk 589 - Guseva et al. (1998) point out the presence of broad He II $\lambda 4686$ and several $\mathrm{N}$ lines in the blue bump, as well as broad $\mathrm{C}$ IV $\lambda 5808$. The $\mathrm{S} / \mathrm{N}$ in the red appears quite low for its detection.

UM 420 - Broad He II $\lambda 4686$ emission has been found by Izotov \& Thuan (1998). From their reanalysis Guseva et al. (1998) signal also nebular He II and broad $\mathrm{C}$ IV $\lambda 5808$. The $\mathrm{S} / \mathrm{N}$ appears fairly low for the latter assertion.

Mrk 1039 - The presence of nebular and broad He II $\lambda 4686$ and C IV $\lambda 5808$ has been discovered by Huang et al. (1998) in this HiI galaxy.

Tol 0226 - 390 - Masegosa et al. (1991) and Pindao (1998) signal the presence of a broad WR bump in this galaxy. 
Table 1. List of WR galaxies (all WR populations outside Local Group). (C91) stands for objects included in Conti (1991) catalogue

\begin{tabular}{|c|c|c|c|c|c|c|c|c|}
\hline Name & $\begin{array}{l}\text { R.A. } \\
{[1950]}\end{array}$ & $\begin{array}{l}\text { Decl. } \\
{[1950]}\end{array}$ & Morphology & $\begin{array}{r}m_{\mathrm{b}} \\
{[\mathrm{mag}]}\end{array}$ & $\begin{array}{r}\begin{array}{r}V_{\text {rad }} \\
{\left[\mathrm{km} \mathrm{s}^{-1}\right]}\end{array} \\
\end{array}$ & $\begin{array}{l}\text { Broad He II } \lambda 4686 \\
\text { reference }\end{array}$ & $\begin{array}{l}\text { Nebular } \\
\text { He II } \lambda 4686\end{array}$ & $\begin{array}{l}\text { C IV } \lambda 5808 \\
\text { reference }\end{array}$ \\
\hline NGC 53 & $0012 \quad 16.1$ & -603619 & $\left(\mathrm{R}^{\prime}\right) \mathrm{SB}(\mathrm{r}) \mathrm{ab}$ & 13.3 & 4568 & MMO91 & & \\
\hline NGC 55 & 001238.0 & -392954 & $\mathrm{SB}(\mathrm{s}) \mathrm{m}: \mathrm{sp}$ & 8.4 & 129 & RD86 & & RD86 \\
\hline UM 228 & 001827.2 & +003609 & & 17.0 & 29490 & MMO91, P98 & & \\
\hline UM 48 & 003335.8 & +042137 & $\mathrm{~S}$ & 15.5 & 4907 & MMO91 & & \\
\hline Mrk 960 & 004604.8 & -125921 & (R)SB0 pec? & 13.5 & 6407 & KC98 & - & \\
\hline NGC 300 & 005231.7 & -375715 & $\mathrm{SA}(\mathrm{s}) \mathrm{d}$ & 8.7 & 144 & DRW83 & & DRW83 \\
\hline IRAS $01003-2238$ & 010023.6 & -223803 & & 18.9 & 35310 & AHM88, (C91) & & \\
\hline UM 311 & 011300.5 & -010722 & & & 1798 & MMO91 & $!$ & IT 98 \\
\hline Tol $0121-376$ & 012155.8 & -373755 & & & 10500 & MMO91, P98 & & \\
\hline Mink & $\begin{array}{lll}01 & 23 & 14.2\end{array}$ & -013754 & Irr & 17.0 & 5638 & $\mathrm{~B} 85,(\mathrm{C} 91)$ & $\mathrm{UL}$ & \\
\hline Mrk 996 & 012504.5 & -063508 & & 15.5 & 1622 & TIL96 & - & TIL96 \\
\hline Mrk 589 & 021108.7 & +035208 & $\mathrm{~S} ?$ & 14.5 & 3436 & GIT98 & - & GIT98 \\
\hline UM 420 & 021820.4 & +001942 & Compact & 16.5 & 17652 & IT98 & $!$ & GIT98 \\
\hline Mrk 1039 & 022507.2 & -102319 & Sc? sp; HII & 15.5 & 2089 & H98 & $\mathrm{Q}$ & H98 \\
\hline Tol $0226-390$ & 022610.0 & -390239 & & 15.3 & 14340 & MMO91, P98 & & \\
\hline Tol $0242-387$ & 024239.2 & -384731 & HII & 17.8 & 37807 & MMO91, P98 & & \\
\hline Mrk 598 & 024352.2 & +07 1134 & & 17.0 & 5396 & P98 & & \\
\hline NGC 1140 & 025208.0 & -101349 & IBm pec & 12.8 & 1501 & GIT98 & - & GIT98 \\
\hline NGC 1156 & 025646.8 & +250221 & $\mathrm{IB}(\mathrm{s}) \mathrm{m}$ & 12.3 & 375 & HFW95 & & \\
\hline NGC 1313 & 031739.0 & -664042 & $\mathrm{SB}(\mathrm{s}) \mathrm{d}$ & 9.2 & 475 & WR97 & & WR97 \\
\hline NGC 1365 & 033141.8 & -361827 & $\left(\mathrm{R}^{\prime}\right) \mathrm{SBb}(\mathrm{s}) \mathrm{b}$ & 10.3 & 1636 & & & PC92 \\
\hline SBS $0335-052$ & 033515.1 & -051226 & & 17.0 & 4043 & I98 & $!$ & \\
\hline NGC 1510 & 040153.9 & -433214 & SA0 pec? & 13.5 & 913 & EN84, (C91) & & \\
\hline NGC 1569 & 042604.6 & +644423 & $\mathrm{IBm}$ & 11.9 & -104 & DR94 & & \\
\hline NGC 1614 & 043135.5 & -084056 & $\mathrm{SB}(\mathrm{s}) \mathrm{c}$ pec & 13.6 & 4778 & P98, (C91) & & \\
\hline VII Zw 19 & 043541.3 & +673819 & & 16.0 & 4830 & KJ85, (C91) & & \\
\hline NGC 1741 (B) & 045906.5 & -042008 & $\mathrm{SB}(\mathrm{s}) \mathrm{m}$ pec & 15.2 & 4171 & KS86, (C91) & $?$ & \\
\hline $\mathrm{H} 31 \mathrm{~A}$ & 045909.9 & -041952 & $\mathrm{Sdm}$ & 15.6 & 4042 & R90, (C91) & & \\
\hline Mrk 1094 & 050817.4 & -024433 & I0 pec? & 14.1 & 2831 & $\mathrm{KJ} 85$ & & \\
\hline II Zw 40 & 055304.9 & +032307 & BCD;Irr & 15.5 & 789 & KS81, (C91) & $!$ & GIT98 \\
\hline Tol $0633-415$ & 063335.0 & $-4131 \quad 12$ & & 16.5 & 5096 & MMO91 & & \\
\hline Mrk 5 & 063524.4 & +754022 & $\mathrm{I} ?$ & 15.6 & 792 & IT98 & & \\
\hline IRAS $07164+5301$ & 071628.6 & +530106 & & & & H96 & & \\
\hline Mrk 1199 & 072028.3 & +333221 & $\mathrm{Sc}$ & 13.7 & 4107 & IT98 & $\mathrm{Q}$ & IT98 \\
\hline NGC 2363 & 072323.7 & +69 1733 & Sm; HII & 11.6 & 107 & G94 & $\mathrm{Q}$ & G94 \\
\hline Mrk 8 & 072336.8 & +721358 & $\mathrm{~S} ?$ & 13.8 & 3496 & KJ85, (C91) & & \\
\hline NGC 2403 & 073205.5 & +654240 & $\mathrm{SAB}(\mathrm{s}) \mathrm{cd}$ & 8.93 & 131 & DR96 & & DR96 \\
\hline VII Zw 187 & 074927.4 & +722457 & & 17.4 & & KJ85 & & \\
\hline SBS $0749+582$ & 074948.0 & +581600 & & 19.0 & 9548 & ITL97 & - & \\
\hline Mrk 1210 & 080127.0 & +051522 & $\mathrm{~S} ?$ & 14.3 & 4046 & SCS98 & $!$ & \\
\hline IRAS $08208+2816$ & 082052.2 & +281557 & Irr & 15.5 & 14034 & H98 & & H98 \\
\hline He $2-10$ & 083407.0 & -261406 & I0? pec & 12.4 & 873 & AWG76, (C91) & & VC92 \\
\hline Mrk 702 & 084245.1 & +161644 & Compact & 15.7 & 15840 & MMO91 & - & GIT98 \\
\hline SBS $0907+543$ & 090731.8 & +542308 & & 17.0 & 8124 & ITL97 & $\mathrm{Q}$ & \\
\hline SBS $0926+606$ & 092620.0 & +604002 & $\mathrm{BCG}$ & 17.5 & 4122 & ITL97 & $\mathrm{Q}$ & \\
\hline I Zw 18 & 093030.2 & +552749 & Compact & 15.6 & 742 & I97 & $\mathrm{Q}$ & I97, L97 \\
\hline ESO $566-7$ & 094238.0 & -192942 & $\mathrm{SBb}$ pec? & 15.3 & 9890 & MMO91 & & \\
\hline NGC 3003 & 094538.5 & +33 3919 & Sbc? & 12.3 & 1478 & HFW95 & & \\
\hline Mrk 22 & 094603.3 & +554848 & & 15.7 & 1499 & ITL94 & $\mathrm{Q}$ & GIT98 \\
\hline Mrk 1236 & 094719.9 & +00 5101 & SABcd & 13.5 & 1829 & KS86, (C91) & $!$ & GIT98 \\
\hline SBS $0948+532$ & $0948 \quad 10.2$ & +531341 & & 18.0 & 13890 & ITL94 & $\mathrm{Q}$ & GIT98 \\
\hline
\end{tabular}


Table 1. continued

\begin{tabular}{|c|c|c|c|c|c|c|c|c|}
\hline Name & $\begin{array}{l}\text { R.A. } \\
{[1950]}\end{array}$ & $\begin{array}{l}\text { Decl. } \\
{[1950]}\end{array}$ & Morphology & $\begin{array}{r}m_{\mathrm{b}} \\
{[\mathrm{mag}]}\end{array}$ & $\begin{array}{r}V_{\text {rad }} \\
{\left[\mathrm{km} \mathrm{s}^{-1}\right]}\end{array}$ & $\begin{array}{l}\text { Broad He II } \lambda 4686 \\
\text { reference }\end{array}$ & $\begin{array}{l}\text { Nebular } \\
\text { He II } \lambda 4686\end{array}$ & $\begin{array}{l}\text { C IV } \lambda 5808 \\
\text { reference }\end{array}$ \\
\hline NGC 3049 & 095210.2 & +093032 & $\mathrm{SB}(\mathrm{rs}) \mathrm{ab}$ & 13.0 & 1494 & KS86, (C91) & - & SCK99 \\
\hline Mrk 712 & 095359.1 & +155234 & $\mathrm{SB}$ & 14.5 & 4560 & CDS95 & $?$ & \\
\hline Tol $0957-278$ & 095705.0 & -275330 & Multiple? & 14.4 & 710 & KJ85 & & \\
\hline NGC 3125 & 100418.2 & -294129 & S & 13.5 & 865 & KS81, (C91) & $?$ & SCK99 \\
\hline Tol $1025-284$ & 102512.0 & -282600 & & 16.9 & 9593 & P98 & & \\
\hline Mrk 33 & 102922.7 & +543931 & Im pec & 13.4 & 1461 & KJ85, (C91) & & \\
\hline Mrk 1434 & 103056.3 & +581920 & $\mathrm{BCG}$ & 16.5 & 2269 & ITL97 & Q & GIT98 \\
\hline Mrk 1259 & 103603.0 & -065437 & S0 pec? & 13.5 & 2159 & OTT97 & - & GIT98 \\
\hline Mrk 724 & 103826.8 & +213724 & Compact & 16.5 & 1139 & KS86, (C91) & $!$ & $\mathrm{KS} 86$ \\
\hline NGC 3353 & 104215.8 & +561326 & $\mathrm{BCD} / \mathrm{Irr}$ & 13.2 & 944 & S96, H98 & $\mathrm{Q}$ & S96, H98 \\
\hline NGC 3367 & 104355.4 & +140058 & $\mathrm{SB}(\mathrm{rs}) \mathrm{c}$ & 12.1 & 3037 & HFW95 & & \\
\hline NGC 3395 & 104702.7 & +331444 & $\mathrm{SAB}(\mathrm{rs}) \mathrm{cd}$ & 12.4 & 1625 & W98 & - & W98 \\
\hline NGC 3396 & 104708.9 & +331518 & IBm pec & 12.6 & 1625 & W98 & $!$ & W98 \\
\hline Mrk 1271 & 105333.3 & +062624 & Compact & 14.8 & 1049 & IT98 & Q & ?GIT98 \\
\hline SBS $1054+365$ & 105459.8 & +363130 & & 16.0 & 603 & ITL97 & - & - \\
\hline Mrk 36 & 110215.6 & +292434 & $\mathrm{BCD}$ & 15.5 & 646 & IT98 & $\mathrm{Q}$ & \\
\hline NGC 3690 & 112542.4 & +585017 & $\mathrm{IBm}$ pec & 12.0 & 3121 & HFW95 & & \\
\hline Mrk 178 & 113045.9 & +493052 & pair & & 249 & GRZ88, (C91) & $!$ & GIT98 \\
\hline NGC 3738 & 113304.2 & +544804 & $\operatorname{Im}$ & 12.1 & 229 & M97 & & \\
\hline UM 439 & 113403.0 & +010536 & & 15.1 & 1169 & MMO91 & & \\
\hline Mrk 182 & 113417.7 & +201212 & Compact & 17.0 & 6328 & GIT98 & $?$ & -- \\
\hline Mrk 1450 & 113551.3 & +580904 & Compact & 15.5 & 946 & ITL94 & Q & ITL94 \\
\hline Mrk 1304 & 113938.6 & +003642 & $\mathrm{Sb}$ pec & 14.7 & 5488 & MMO91 & $!$ & GIT98 \\
\hline Mrk 1305 & 114024.6 & -080318 & & 15.5 & 2997 & GIT98 & - & GIT98 \\
\hline Mrk 750 & 114728.0 & +151804 & BCD & 15.8 & 754 & KJ85, (C91) & Q & GIT98 \\
\hline Pox 4 & 114839.0 & -201917 & & 16.2 & 3589 & KJ85 & $?$ & \\
\hline UM 461 & 114859.4 & -020541 & $\mathrm{BCD} / \mathrm{Irr}$ & 16.9 & 899 & C91 & & \\
\hline Mrk 1307 & 115003.8 & -021128 & Pec;BCD & 14.5 & 1012 & IT98 & Q & \\
\hline Mrk 193 & 115252.1 & +575626 & Compact & 16.5 & 5282 & GIT98 & $!$ & \\
\hline ISZ 59 & 115454.7 & -192044 & S0 & 16.8 & 1781 & KJ85, (C91) & & \\
\hline NGC 3995 & 115510.4 & +323424 & SAm pec & 12.7 & 3254 & W98 & $!$ & W98 \\
\hline NGC 4038 & 115919.0 & -183512 & $\mathrm{SB}(\mathrm{s}) \mathrm{m}$ pec & 10.9 & 1642 & RD86 & & \\
\hline SBS $1211+540$ & 121133.9 & +540158 & & 17.8 & 929 & GIT98 & $!$ & \\
\hline NGC 4214 & 121308.3 & +363622 & $\mathrm{IAB}(\mathrm{s}) \mathrm{m}$ & 10.2 & 291 & SF91 & & SF91, MHK91 \\
\hline NGC 4216 & 121321.5 & +132540 & $\mathrm{SAB}(\mathrm{s}) \mathrm{b}$ & 11.0 & 131 & $\mathrm{RD} 86$ & & \\
\hline NGC 4236 & 121421.8 & +694436 & $\mathrm{SB}(\mathrm{s}) \mathrm{dm}$ & 10.1 & 0 & GP94 & & \\
\hline M 106 & 121629.4 & +473453 & $\mathrm{SAB}(\mathrm{s}) \mathrm{bc}$ & 9.10 & 448 & C98 & & \\
\hline SBS $1222+614$ & 122244.5 & +612546 & & 17.0 & 734 & ITL97 & $\mathrm{Q}$ & ITL97 \\
\hline NGC 4385 & 122309.0 & +005057 & $\mathrm{SB}(\mathrm{rs}) 0+$ & 13.2 & 2140 & CS86, (C91) & & CS86 \\
\hline II Zw 62 & 122338.4 & +075637 & & 17.2 & 3930 & KJ85, (C91) & & \\
\hline Mrk 209 & 122350.5 & +484613 & Sm pec & 15.2 & 281 & ITL97 & $\mathrm{Q}$ & GIT98 \\
\hline NGC 4449 & 122545.9 & +442216 & $\mathrm{IBm}$ & 10.0 & 207 & MK97 & & MK97 \\
\hline NGC 4532 & 123146.7 & +064439 & $\mathrm{IBm}$ & 12.3 & 2012 & HFW95 & & \\
\hline Mrk 1329 & 123429.8 & +071201 & SBmIII & 14.4 & 1632 & GIT98 & - & ?GIT98 \\
\hline Tol $1235-350$ & 123546.0 & -350242 & S0: & 17.1 & 2998 & P98 & & \\
\hline NGC 4670 & 124249.8 & +272354 & $\mathrm{SB}(\mathrm{s}) 0 / \mathrm{a}$ pec & 13.1 & 1069 & MHK91 & & \\
\hline Tol $1247-232$ & 124739.0 & -231738 & & 15.5 & 14400 & MMO91 & & \\
\hline SBS $1249+493$ & 124935.8 & +491945 & $\mathrm{BCG}$ & 17.5 & 7330 & GIT98 & $!$ & \\
\hline NGC 4861 & 125639.7 & +350750 & $\mathrm{SB}(\mathrm{s}) \mathrm{m}$ & 12.9 & 847 & DS86, (C91) & $\mathrm{Q}$ & DS86 \\
\hline Tol 30 & 130303.0 & -280912 & $\mathrm{SB}$ & 15.5 & & C96 & $?$ & \\
\hline Pox 120 & 130404.7 & -114820 & & 15.7 & 6220 & KJ85, (C91) & & \\
\hline
\end{tabular}


Table 1. continued

\begin{tabular}{|c|c|c|c|c|c|c|c|c|}
\hline Name & $\begin{array}{l}\text { R.A. } \\
{[1950]}\end{array}$ & $\begin{array}{l}\text { Decl. } \\
{[1950]}\end{array}$ & Morphology & $\begin{array}{r}m_{\mathrm{b}} \\
{[\mathrm{mag}]}\end{array}$ & $\begin{array}{r}V_{\text {rad }} \\
{\left[\mathrm{km} \mathrm{s}^{-1}\right]}\end{array}$ & $\begin{array}{l}\text { Broad He II } \lambda 4686 \\
\text { reference }\end{array}$ & $\begin{array}{l}\text { Nebular } \\
\text { He II } \lambda 4686\end{array}$ & $\begin{array}{l}\text { C IV } \lambda 5808 \\
\text { reference }\end{array}$ \\
\hline Pox 139 & 130920.8 & -114754 & $\mathrm{SB}(\mathrm{s}) \mathrm{d}$ & 15.0 & 2107 & KJ85, (C91) & & \\
\hline NGC 5068 & 131613.0 & -204636 & $\mathrm{SB}(\mathrm{s}) \mathrm{d}$ & 10.7 & 673 & $\mathrm{RD} 86$ & & \\
\hline SBS $1319+579$ & 131925.2 & +575709 & & 18.5 & 2060 & ITL97 & $\mathrm{Q}$ & GIT98 \\
\hline NGC 5128 & 132231.6 & -424533 & S0 pec & 7.8 & 547 & M 81, RD86 & & M 81 \\
\hline Pox 186 & 132312.0 & -112200 & & 17.0 & 1170 & KJ85, (C91) & & \\
\hline Tol 35 & 132420.0 & -274148 & $\mathrm{~S} ?$ & 14.4 & 1814 & KJ85, (C91) & $?$ & \\
\hline M 83 & 133411.5 & -293642 & $\mathrm{SAB}(\mathrm{s}) \mathrm{c}$ & 8.2 & 516 & RD86 & & \\
\hline NGC 5253 & 133705.1 & -312313 & IM pec & 10.9 & 404 & CTM86, (C91) & $?$ & SCK99 \\
\hline Mrk 67 & 133939.6 & +304616 & BCD/Irr & 16.5 & 958 & C91 & & \\
\hline Mrk 1486 & 135809.4 & +574054 & & & 10143 & ITL97 & Q & \\
\hline Tol 89 & 135826.0 & -324920 & $\mathrm{SB}(\mathrm{rs}) \mathrm{dm}$ & 16.0 & 1216 & DBB85, (C91) & $?$ & SCK99 \\
\hline NGC 5430 & 135908.5 & +593416 & $\mathrm{SB}(\mathrm{s}) \mathrm{b}$ & 12.7 & 3028 & K82, (C91) & & \\
\hline NGC 5408 & 140017.5 & -410819 & $\mathrm{IB}(\mathrm{s}) \mathrm{m}$ & 12.2 & 509 & MMO91 & $!$ & \\
\hline NGC 5457 & 140126.3 & +543518 & $\mathrm{SAB}(\mathrm{rs}) \mathrm{cd}$ & 8.3 & 241 & R82, DRW83 & & \\
\hline NGC 5461 & 140154.9 & +543324 & & & 298 & $\mathrm{R} 82$ & & \\
\hline NGC 5471 & 140243.4 & +543808 & & 15.0 & 297 & MHK91 & $?$ & \\
\hline SBS $1408+551 \mathrm{~A}$ & 140814.0 & +551100 & & 18.0 & 23190 & I96 & $\mathrm{Q}$ & I96 \\
\hline CGCG 219-066 & 141503.6 & +434404 & & 15.6 & 649 & I98 & $!$ & \\
\hline Mrk 475 & 143703.6 & +370113 & $\mathrm{BCD}$ & 14.5 & 540 & $\mathrm{C} 91$ & $\mathrm{Q}$ & ITL94 \\
\hline Mrk 477 & 143902.5 & +534304 & & & 11340 & H97 & $!$ & \\
\hline Tol 1457-262A & 145731.8 & -261440 & HII & & 5180 & C96 & - & \\
\hline Tol 1457-262B & 145732.1 & -261449 & HII & 14.7 & 5249 & P98 & $?$ & \\
\hline SBS $1533+574 B$ & 153304.1 & +572700 & & & 3390 & GIT98 & $!$ & GIT98 \\
\hline IC 4662 & 174212.0 & -643718 & $\mathrm{IBm}$ & 11.7 & 308 & RD86 & & RR91 \\
\hline NGC 6500 & 175348.1 & +182041 & SAab & 13.1 & 3003 & B97 & & \\
\hline Fairall 44 & 180919.3 & -574448 & S? pec & 14.8 & 4948 & KC98 & $\mathrm{Q}$ & КC98 \\
\hline NGC 6764 & 190701.2 & +505108 & $\mathrm{SB}(\mathrm{s}) \mathrm{bc}$ & 12.6 & 2416 & OC82, (C91) & & \\
\hline Tol 1924-416 & 192429.0 & -414036 & pec HII & 13.3 & 2874 & $\mathrm{P} 98$ & $?$ & \\
\hline IC 4870 & 193248.0 & -655530 & IBm? pec Sy2 & 13.9 & 889 & JK99 & $!$ & \\
\hline IC 5154 & 220041.7 & -662125 & Irr Sy2 & 14.9 & 3118 & JK99 & $?$ & JK99 \\
\hline ESO 108-IG 017 & 220700.3 & -670659 & I0? Sy2 & 14.4 & 2191 & JK99 & $?$ & JK99 \\
\hline Mrk 309 & 225010.0 & +242752 & $\mathrm{Sa}$ & 15.4 & 12645 & OC82, (C91) & & OC82 \\
\hline Mrk 315 & 230135.7 & +222116 & E1 pec? & 14.8 & 11661 & KJ85 & & \\
\hline ESO 148-IG 02 & 231251.0 & -591940 & Merger & 15.2 & 13380 & JB88, (C91) & & \\
\hline III Zw 107 & 232740.4 & +251527 & $\mathrm{Sb}$ & 15.6 & 6176 & KJ85, (C91) & & \\
\hline Mrk 930 & 232929.2 & +284016 & & & 5400 & IT98 & $!$ & GIT98 \\
\hline NGC 7714 & 233340.6 & +015242 & $\mathrm{SB}(\mathrm{s}) \mathrm{b}$ pec & 13.0 & 2798 & $\mathrm{~B} 85,(\mathrm{C} 91)$ & $\mathrm{Q}$ & G97 \\
\hline
\end{tabular}

Key to Col. 8 (nebular He II). - no entry: no information available, "-": absent, "?": possible/suspected, "!": present, but no data available, "UL": upper limit available, "Q": measurement available. Key to references. - AHM88 Armus et al. (1988), AWG76 Allen et al. (1976), B85 Breugel et al. (1985), B97 Barth et al. (1997), C91 Conti (1991), C96 Contini (1996), C98 Castellanos et al. (1998), CDS95 Contini et al. (1995), CS86 Campbell \& Smith (1986), CTM86 Campbell et al. (1986), DBB85 Durret et al. (1985), DR94 Drissen \& Roy (1994), DR96 Drissen \& Roy (1996), DRM93 Drissen et al. (1993), DRW83 D'Odorico et al. (1983), DS86 Dinerstein \& shields (1986), EN84 Eichendorf \& Nieto (1984), G97 García-Vargas et al. (1997), GIT98 Guseva et al. (1998), GP94 Gonzalez-Delgado \& Perez (1994), GRZ88 Gonzalez-Riestra et al. (1988), H96 Huang et al. (1996), H97 Heckman et al. (1997), H98 Huang et al. (1998), HFW95 Ho et al. (1995), I96 Izotov et al. (1996), I97 Izotov et al. (1997b), I98 Izotov et al. (1998), IT98 Izotov \& Thuan (1998), ITL94 Izotov et al. (1994), ITL97 Izotov et al. (1997a), JB88 Johansson \& Bergvall (1988), JK99 Joguet \& Kunth (1999), K82 Keel (1982), KC98 Kovo \& Contini (1998), KJ85 Kunth \& Joubert (1985), KS81 Kunth \& Sargent (1981), KS86 Kunth \& Schild (1986), KMH94 Kunth \& Mas-Hesse (1994), L97 Legrand et al. (1997), M 81 Moellenhoff (1981), M97 Martin (1997), MK97 Martin \& Kennicut (1997), MMO91 Masegosa et al. (1991), MHK91 MasHesse \& Kunth (1991), OC82 Osterbrock \& Cohen (1982), OTT97 Ohyama et al. (1997), P98 Pindao (1998), PC92 Phillips \& Conti (1992), R82 Rayo et al. (1982), RR91 Richter \& Rosa (1991), R90 Rubin et al. (1990), RD86 Rosa \& D’Odorico (1986), S97 Schaerer et al. (1997), S96 Steel et al. (1996), SCK99 Schaerer et al. (1999), SCS98 Storchi-Bergmann et al. (1998), SF91 Sargent \& Fillipenko (1991), TIL96 Thuan et al. (1996), V88 Vilchez et al. (1988), V96 Vacca (1996), VC92 Vacca \& Conti (1992), W98 Weistrop et al. (1998), WR97 Walsh \& Roy (1997). 
Table 2. List of extragalactic HiI regions with nebular He II

\begin{tabular}{|c|c|c|c|c|c|c|c|}
\hline Name & $\begin{array}{l}\text { R.A. } \\
{[1950]}\end{array}$ & $\begin{array}{l}\text { Decl. } \\
{[1950]}\end{array}$ & "Morphology & $\begin{array}{r}m_{\mathrm{b}} \\
{[\mathrm{mag}]}\end{array}$ & $\begin{array}{r}V_{\mathrm{rad}} \\
{\left[\mathrm{km} \mathrm{s}^{-1}\right]}\end{array}$ & $\begin{array}{l}\begin{array}{l}\text { Nebular He II } \lambda 4686 \\
\text { reference }\end{array}\end{array}$ & Comment \\
\hline SBS $0749+568$ & 074937.7 & +564948 & BCG & 18.0 & 5471 & ITL97 & \\
\hline Mrk 1416 & 091725.9 & +524650 & Irr & 17.0 & 2305 & ITL97 & \\
\hline Tol 4 & 100831.0 & -283918 & $\mathrm{E}$ ? & 14.4 & 4219 & CTM86 & also C96; P99: WR? \\
\hline SBS $1116+583 B$ & 111631.4 & +582016 & & 19.5 & 9905 & ITL97 & \\
\hline UM 469 & 115438.6 & +024510 & & 18.0 & 17388 & C96 & \\
\hline SBS $1159+545$ & 115929.0 & +543233 & BCG & 18.0 & 3537 & ITL94 & \\
\hline SBS $1205+557$ & 120557.5 & +554207 & $\mathrm{BCG}$ & 15.5 & 1751 & ITL97 & \\
\hline Tol 21 & 121442.0 & -274500 & & 17.5 & 7795 & CTM86 & \\
\hline Mrk 1318 & 121636.5 & +040758 & E pec & 14.0 & 1526 & C96 & P99: WR? \\
\hline Fairall 30 & 123632.0 & -395442 & $\mathrm{SAB}(\mathrm{r}) 0$ & & 1199 & CTM86 & \\
\hline UM 533 & 125725.0 & +021908 & dIn & 15.7 & 874 & KC98 & \\
\hline Pox 105 & 130000.0 & -111000 & & 17.0 & 3405 & KS83, KJ85 & \\
\hline Tol $1304-386$ & 130432.0 & -383848 & & & 4197 & CTM86 & P99: WR? \\
\hline Tol 78 & 130448.0 & -352200 & & 17.0 & 4197 & CTM86 & \\
\hline Tol 111 & 134524.0 & -420600 & & 16.3 & 2398 & CTM86 & \\
\hline SBS $1420+544$ & 142059.1 & +542742 & BCG & 18.0 & 6176 & TIL95 & \\
\hline CG 1258 & 144150.9 & +292832 & & 18.0 & 13614 & ITL97 & \\
\hline SBS $1533+469$ & 153324.0 & +465900 & & & 5666 & TIL95 & \\
\hline HS $1851+6933$ & 185138.6 & +693316 & & 17.0 & 7495 & I96 & \\
\hline
\end{tabular}

Key to references. - C96 Contini (1996), CTM86 Campbell et al. (1986), I96 Izotov et al. (1996), I97c Izotov et al. (1997c), ITL94 Izotov et al. (1994), ITL97 Izotov et al. (1997a), KC98 Kovo \& Contini (1998), KJ85 Kunth \& Joubert (1985), KS83 Kunth \& Sargent (1983), P98 Pindao (1998), P99 Pindao et al. (1999), TIL Thuan et al. (1995).

Tol 0242-387 — Masegosa et al. (1991) and Pindao (1998) signal the presence of a broad WR bump in this galaxy.

Mrk 598 - Pindao (1998) signals the presence of a broad WR bump in the western knot of this galaxy.

NGC 1140 - A broad WR bump and C IV $\lambda 5808$ are detected by Guseva et al. (1998).

NGC 1156 - Emission features of WR stars and a high-excitation HII region in the nucleus are signaled by Ho et al. (1995) from their magnitude limited survey of nuclei of nearby galaxies. A close resemblance of the spectrum with NGC 4214 is pointed out. They also signal a broad $\mathrm{H} \alpha$ component.

NGC 1313 - WR features are found in two regions at large galactocentric radii of this Transition Magellanic galaxy (Walsh \& Roy 1997). For region \# 28 both He II $\lambda 4686$ and C IV $\lambda 5808$ are found. No detailed information is provided about region \# 3. Pindao et al. (1999) also signal the possible detection of broad He II $\lambda 4686$; exact position unknown.

NGC 1365 - The detection of broad C III $\lambda 5696$ and $\mathrm{C}$ IV $\lambda 5808$ (marginally) in this giant extragalactic HII region was made by Phillips \& Conti (1992).

SBS 0335-052 - The first detection of nebular He II $\lambda 4686$ in this very low metallicity object was reported by Izotov et al. (1990). Nebular and broad He II $\lambda 4686$ have been found in the reanalysis of Izotov et al. (1998); no broad features had been signalled by Izotov et al. (1997c).
NGC 1510 - Eichendorf \& Nieto (1984) show the presence of broad He II $\lambda 4686$ in one component of this amorphous galaxy. See the discussion in Conti (1991) for more details.

NGC 1569 - Narrow-band $\lambda 4686$ filter imaging of Drissen et al. (1993) revealed the possible presence of WR stars. Spectroscopy by Drissen \& Roy (1994) in the outskirts of the galaxy shows broad He I $\lambda 5876$ and He I $\lambda 6678$ both attributed to a late WN star. According to GonzalézDelgado et al. (1997) this region is located $4^{\prime \prime}$ west of super star cluster (SSC) A. Ho et al. (1995) find the WR bump and a broad $\mathrm{H} \alpha$ component "in the nuclear spectrum". Gonzaléz-Delgado et al. (1997) find several broad features in the WR bump which are confined to SSC $A$. Broad He II $\lambda 4686$ has also been detected by Martin \& Kennicutt (1997).

NGC 1614 - A broad WR bump is detected by Pindao (1998). The measurement of Vacca \& Conti (1992) provides an upper limit on broad He II $\lambda 4686$.

VII Zw 19 — Found by Kunth \& Joubert (1985) in their "survey" of 45 blue emission line galaxies for showing excess emission between 4600 and $4711 \AA$.

NGC 1741 - This well studied galaxy is part of the Hickson (1982) "compact" group \# 31 and interacting with H31 (see below). Several broad components in the WR bump (He II $\lambda 4686$, N III $\lambda 4640$ ) and nebular emission of [Fe III] $\lambda 4658$ and He II $\lambda 4686$ have been identified by Kunth \& Schild (1986). According to Conti (1991) 
Table 3. List of suspected WR galaxies

\begin{tabular}{|c|c|c|c|c|c|c|}
\hline Name & $\begin{array}{l}\text { R.A. } \\
{[1950]}\end{array}$ & $\begin{array}{l}\text { Decl. } \\
{[1950]}\end{array}$ & Morphology & $\begin{array}{r}m_{\mathrm{b}} \\
{[\mathrm{mag}]}\end{array}$ & $\begin{array}{r}V_{\text {rad }} \\
{\left[\mathrm{km} \mathrm{s}^{-1}\right]}\end{array}$ & Reference \\
\hline Fairall 4 & 001249.2 & -573122 & (R)SA(r)b & 15.1 & 9796 & P99 \\
\hline Tol $0030-388$ & 003038.3 & -385029 & & & 13800 & MMO91 \\
\hline Fairall 0007 & 004206.0 & -600700 & & & & MMO91 \\
\hline UM 080 & 005746.8 & +042346 & & 17.8 & 4797 & P99 \\
\hline EQ $0102-310$ & 0102 & -31.0 & & & & MMO91 \\
\hline Tol 0127-397 & 012702.4 & -394603 & & & 4797 & P99 \\
\hline UM 354 & 013711.1 & +011057 & & & 9294 & P99 \\
\hline UM 377 & 015218.8 & +010228 & & & 8694 & P99 \\
\hline UM 396 & 020451.0 & +024241 & & & 6296 & P99 \\
\hline NGC 848 & 020750.4 & -103325 & $\left(\mathrm{R}^{\prime}\right) \mathrm{SB}(\mathrm{s}) \mathrm{ab}$ & 13.6 & 4001 & GIT98 \\
\hline NGC 1068 & 024007.0 & -001332 & (R)SA(rs)b & 9.6 & 1136 & ED86 \\
\hline CAM $0357-3915$ & 035722.1 & -391451 & HII & & 22545 & P99 \\
\hline Tol $0440-381$ & 044023.3 & -380641 & & & 12300 & MMO91, P99 \\
\hline Tol $0513-393$ & 051339.7 & -392059 & & & 15000 & P99 \\
\hline Tol $0538-416$ & 053832.9 & -413837 & HII & & 13491 & P99 \\
\hline Tol $0559-393$ & 055904.9 & -391908 & Irr & 15.0 & 24915 & MMO91, P99 \\
\hline Tol $0620-386$ & 063008.3 & -383911 & & & 20100 & P99 \\
\hline Tol $0645-376$ & 064507.0 & -374002 & & & 7795 & MMO91 \\
\hline NPM1G+16.0158 & 084101.4 & +161038 & & 18.3 & & P99 \\
\hline ESO $566-8$ & 094239.2 & -192855 & S pec & 16.3 & 9761 & C96 \\
\hline Mrk 709 & 094633.2 & +170644 & $\mathrm{BCD}$ & 17.0 & 1197 & P99 \\
\hline Tol $1021-289$ & 102122.0 & -285830 & Interacting & 16.9 & 17658 & P99 \\
\hline Tol $1025-285$ & 102506.2 & -283213 & $\mathrm{~N}$ & 16.2 & 8694 & P99 \\
\hline Abell 1068 & 103751.3 & +401251 & $\mathrm{cD}$ & 16.0 & 41580 & A95 \\
\hline Tol $1032-283$ & 103218.4 & -281952 & E4: & 14.4 & 3190 & KS86 \\
\hline Mrk 1301 & 113310.9 & +353643 & $\mathrm{~S} 0 / \mathrm{a}$ & 14.5 & 1603 & RC93 \\
\hline UM 456 & 114802.5 & -001721 & Pec & 15.9 & 1757 & P99 \\
\hline Pox 52 & 120022.9 & -203921 & & 17.2 & & KSB87 \\
\hline UM 482 & 120929.4 & -001943 & $\mathrm{Sm}$ & 16.2 & 10544 & P99 \\
\hline UM 483 & 120941.0 & +002101 & & 16.7 & 2099 & P99 \\
\hline M100 & 122022.9 & +160558 & $\mathrm{SAB}(\mathrm{s}) \mathrm{bc}$ & 7.4 & 1571 & WO98 \\
\hline NGC 4509 & 123038.9 & +322202 & Sab pec? & 14.1 & 937 & RC93 \\
\hline Tol $1258-363$ & 125819.0 & -361954 & & & 4887 & P99 \\
\hline UM 594 & 133532.7 & +001626 & HII? & 15.8 & 6653 & P99 \\
\hline NGC $5257(\mathrm{~W})$ & 133719.7 & +010533 & $\mathrm{SAB}(\mathrm{s}) \mathrm{b}$ pec & 12.9 & 6798 & MMO91 \\
\hline NGC 5291 & 134433.3 & -300931 & E pec: & 15.1 & 4386 & DM98 \\
\hline Tol $1345-419$ & 134514.2 & -415523 & Sy2 & 17.0 & 11572 & P99 \\
\hline UM 618 & 135003.1 & +001607 & & 18.4 & 4197 & P99 \\
\hline Abell 1835 & 135830.0 & +030600 & $\mathrm{cD}$ & 19.3 & 75690 & A95 \\
\hline EQ $1409-120$ & 1409 & +0120 & & & & MMO91 \\
\hline Cam $1409+1200$ & 140914.3 & +115933 & & & 16800 & P99 \\
\hline NGC 6090 & 161024.0 & +523504 & Sd pec & 14.5 & 8822 & RC93 \\
\hline Tol $2006-393$ & 200639.7 & -392232 & & & 9294 & P99 \\
\hline Tol $2138-405$ & 213814.8 & -403235 & & & 16800 & P99 \\
\hline Tol $2146-391$ & 214645.0 & -390810 & & & 8994 & MMO91, P99 \\
\hline Tol $2259-398$ & 225934.0 & -394942 & Compact & 15.6 & 8858 & MMO91, P99 \\
\hline UM 159 & 231931.9 & +011224 & HII & & 8694 & P99 \\
\hline UM $160 \mathrm{~W}$ & 232146.0 & -002330 & & & 2400 & P99 \\
\hline
\end{tabular}

Key to references. - A95 Allen (1995), C91 Conti (1991), DM98 Duc \& Mirabel (1998) DRM93 Drissen et al. (1993), ED86 Evans \& Dopita (1986), GIT98 Guseva et al. (1998), KS86 Kunth \& Schild (1986), KSB87 Kunth et al. (1987), MMO91 Masegosa et al. (1991), P99 Pindao et al. (1999), RC93 Robledo-Rella \& Conti (1993), VC92 Vacca \& Conti (1992), WO98 Wozniak et al. (1998). 
N III $\lambda 4640$ cannot be confirmed. The spectrum of Vacca \& Conti (1992) of region B is shown in Conti et al. (1996) who obtained also a UV spectrum with GHRS on HST of this region. An upper limit on He II $\lambda 4686$ is also given by Vacca \& Conti (1992) for region A. The high S/N spectrum of Izotov \& Thuan (1998) shows broad He II $\lambda 4686$, whereas the C IV $\lambda 5808$ is absent. No nebular He II component is present according to the reanalysis of Guseva et al. (1998).

H31A - A broad He II $\lambda 4686$ feature and was pointed out by Rubin et al. (1990) in galaxy A and possibly also in $\mathrm{H} 31 \mathrm{C}=$ NGC 1741 (see above).

Mrk 1094 - Kunth \& Joubert (1985) list this object as having a broad He II $\lambda 4686$ excess above $0.8 \sigma$ of the background. Broad He II $\lambda 4686$ was detected by Vacca \& Conti (1992) in region A. Upper limits on He II are given for two other regions.

II Zw 40 - Broad He II $\lambda 4686$ was detected by Kunth \& Sargent (1981) in this well studied low metallicity galaxy. See also Vacca \& Conti (1992) for more recent observations and a study of its WR content. Broad He II $\lambda 4686$ and possibly also C IV $\lambda 5808$ are signaled by Martin (1997). An important contamination by nebular He II $\lambda 4686$ has been suspected by Schaerer (1996) from its similarity with Pox 4 and on theoretical grounds. A broad asymmetric emission components of $\mathrm{H} \alpha$ has been found by Méndez \& Esteban (1997). Guseva et al. (1998) find the presence of both stellar and nebular He II and broad C IV $\lambda 5808$.

Tol 0633 - 415 - Automatic detection by Masegosa et al. (1991). Pindao et al. (1999) classify this as a suspected WR galaxy.

Mrk 5 - The high $\mathrm{S} / \mathrm{N}$ spectrum of Izotov \& Thuan (1998) shows broad He II $\lambda 4686$. No nebular He II component is present according to the reanalysis of Guseva et al. (1998).

IRAS 07164+5301 - Huang et al. (1996) detect the presence of broad lines around $4686 \AA$ suggesting N III $\lambda 4640, \mathrm{C}$ III $\lambda 4650$, and He II $\lambda 4686$ in this IRAS source. They also indicate a tentative detection of $\mathrm{O} v \lambda 5835$ and a lack of C IV $\lambda 5808$. The spectrum is of fairly low $\mathrm{S} / \mathrm{N}$.

Mrk 1199 - Izotov \& Thuan (1998) signal nebular and broad He II $\lambda 4686$ and C IV $\lambda 5808$ features from their high $\mathrm{S} / \mathrm{N}$ spectrum ${ }^{2}$. The latter features appears quite weakly. No nebular He II component is present according to the reanalysis of Guseva et al. (1998).

NGC 2363 - This is a well studied giant HII region consisting of two main knots and located south west of the irregular galaxy NGC 2366 (e.g. Drissen et al. 1993; Gonzaléz-Delgado et al. 1994, and references therein). Spectra of the region taken up to 1992 all show narrow

\footnotetext{
${ }^{2}$ The reported nebular He II $\lambda 4686$ intensity in Izotov \& Thuan (1998) is erroneous according to Y. Itozov (1998, private commnication). No indication is therefore given in Col. 8 of Table 1 for this object.
}

He II $\lambda 4686$ (see references in Drissen et al. 1993). From narrow-band imaging Drissen et al. (1993) find excess He II $\lambda 4686$ emission in both knots, but much stronger in the fainter eastern knot. They argue for WR stars in this knot (B). Spectroscopy by Gonzaléz-Delgado et al. (1994) confirms the presence broad and nebular He II $\lambda 4686$ in knot B, and detect also C IV $\lambda 5808$ attributed to WC stars. Izotov et al. (1997a) find broad and nebular He II $\lambda 4686$ emission in knot A, and nebular He II in knot B (cf. also the reanalysis by Guseva et al. 1998). Broad emission component of $\mathrm{H} \alpha, \mathrm{H} \beta$, and [O III] are also known (Roy et al. 1992; Izotov et al. 1997a).

Mrk 8 - Kunth \& Joubert (1985) list this object as having a broad He II $\lambda 4686$ excess above $0.8 \sigma$ of the background.

NGC 2403 - Drissen \& Roy (1996) detect broad He II $\lambda 4686$ and C IV $\lambda 5808$ in two giant HII regions in this galaxy of the M 81 group.

VII Zw 187 - Kunth \& Joubert (1985) list this object as having a broad He II $\lambda 4686$ excess above $0.8 \sigma$ of the background.

SBS 0749+582 - Broad and nebular He II $\lambda 4686$ is signaled by Izotov et al. (1997a).

Mrk 1210 - Storchi-Bergmann et al. (1998) detect the presence of a broad He II $\lambda 4686$ component attributed to WR stars in the nucleus of this Seyfert 2 galaxy. A broad WR bump is also detected by Pindao (1998).

IRAS 08208+2816 - The presence of nebular and broad He II $\lambda 4686$ and broad C IV $\lambda 5808$ was found by Huang et al. (1998) in this luminous infrared galaxy. The WR bump luminosity is exceptionally large and rivals that of IRAS $01003-2238$. Interestingly the authors also find essentially zero internal reddening derived from the Balmer decrement.

He 2-10 - This dwarf emission galaxy can be considered the "prototypical WR galaxy" since it was the first where He II $\lambda 4686$ emission attributed to WR stars was detected (Allen et al. 1976). Abundant observational data is available for this galaxy. The detection of Allen et al. has been confirmed by Hutsemekers \& Surdey (1984), who also suspected C IV $\lambda 5808$ emission due to WC stars from their spectrum. Broad He II $\lambda 4686$ and a weak C IV $\lambda 5808$ feature were detected by Vacca \& Conti (1992) in their region $A$. Both features were confirmed by Schaerer et al. (1999) from their high $\mathrm{S} / \mathrm{N}$ spectra. An upper limit for He II $\lambda 4686$ in region B is also given in Vacca \& Conti (1992). The HST UV images of Conti \& Vacca (1994) resolve this galaxy in multiple knots. Broad asymmetric emission components of $\mathrm{H} \alpha$ and [N II] $\lambda 6584$ have been found by Méndez \& Esteban (1997).

Mrk 702 - An incorrect object name (C 0842+163) was used for this galaxy by Masegosa et al. (1991), who report a broad WR feature. Guseva et al. (1998) detect both broad He II $\lambda 4686$ and C IV $\lambda 5808$ features. The S/N appears fairly low for the latter assertion. 
SBS 0907+543 — Broad and nebular He II $\lambda 4686$ has been found by Izotov et al. (1997a).

SBS 0926+606 — Broad and nebular He II $\lambda 4686$ has been found by Izotov et al. (1997a) (cf. also Guseva et al. 1998). They also indicate the presence of low intensity broad components of $\mathrm{H} \alpha$ and/or [O III] $\lambda 5007$.

I Zw 18 - This well-known object is the galaxy with the lowest metal content known. While nebular He II $\lambda 4686$ was observed for a long time, only recently the deep spectra of Izotov et al. (1997b) and Legrand et al. (1997) revealed several broad emission components (C III $\lambda 4650$, He II $\lambda 4686, \mathrm{C}$ IV $\lambda 5808)$ attributed to WN and WC stars. The spatial distribution of He II $\lambda 4686$ emission was studied by Hunter \& Thronson (1995) and De Mello et al. (1998) based on WFPC2 HST observations. Izotov et al. (1997a) indicate the presence of low intensity broad components of $\mathrm{H} \alpha$ and/or [O III] $\lambda 5007$.

ESO 566 - 7 - An incorrect object name C $0942-$ 1929A was used by Masegosa et al. (1991) for this galaxy, where they report a broad WR feature. Pindao (1998) confirms the presence of the WR bump.

NGC 3003 - A complex broad WR-bump was signaled by Ho et al. (1995), who also note the absence of a broad $\mathrm{H} \alpha$ component in contrast to their spectra of other WR galaxies (cf. NGC 1156, NGC 1569, NGC 4214).

Mrk 22 - Broad and nebular He II $\lambda 4686$ has been found by Izotov et al. (1994). Their spectrum appears noisy to detect $\mathrm{C}$ IV $\lambda 5808$. Its detection is, however, signaled by Guseva et al. (1998).

Mrk 1236 - Kunth \& Schild (1986) point out the presence of broad He II $\lambda 4686$ in this galaxy. Broad He II $\lambda 4686$ was detected in region A by Vacca \& Conti (1992). Observations of Guseva et al. (1998) signal the presence of nebular and broad He II, broad C IV $\lambda 5808$ and possibly also C III $\lambda 5696$. A broad emission feature is identified as C II $\lambda 4267$.

SBS 0948+532 — Broad and nebular He II $\lambda 4686$ has been found by Izotov et al. (1994). Also C IV $\lambda 5808$ is present according to the reanalysis of Guseva et al. (1998), although the $\mathrm{S} / \mathrm{N}$ appears fairly low.

NGC 3049 - This Virgo Cluster galaxy shows broad [N II] $\lambda 5755$ and He II $\lambda 4686$ (Kunth \& Schild 1986). The WR bump is confirmed by Mas-Hesse \& Kunth (1991, 1998), Masegosa et al. (1991), and Pindao (1998). Broad He II $\lambda 4686$ was detected in region $A$ by Vacca \& Conti (1992); an upper limit is given for region $B$. The high $\mathrm{S} / \mathrm{N}$ observations of Schaerer et al. (1999) reveal broad features of N III $\lambda 4640$, He II $\lambda 4686$, C III $\lambda 5696$, and C IV $\lambda 5808$ testifying of the presence of late-type WN and late-type WC stars. These features are confirmed by Guseva et al. (1998).

Mrk 712 - Contini et al. (1995) and Contini (1996) signal the presence of broad N III $\lambda 4640$ and He II $\lambda 4686$ in a giant HII region of this IRAS barred spiral galaxy. A nebular contribution to He II may be present (Contini 1996)
Tol $0957-278$ - Kunth \& Joubert (1985) list this object (=Tol 2) as having a broad He II $\lambda 4686$ excess above $0.8 \sigma$ of the background in the NE component. Upper limits of He II $\lambda 4686$ are given for 2 regions by Vacca \& Conti (1992). Possible detection of broad He II $\lambda 4686$ according to Pindao et al. (1999).

NGC 3125 - Broad He II $\lambda 4686$ emission was found by Kunth \& Sargent (1981; cf. also Kunth \& Joubert 1985) in this dwarf galaxy. They also note narrow [Fe III] $\lambda 4658$ emission. Broad He II $\lambda 4686$ was detected in two regions by Masegosa et al. (1991) and Vacca \& Conti (1992). Pindao (1998) reconfirms the detections of Masegosa et al.. The high S/N observations of Schaerer et al. (1999) reveal broad features of N III $\lambda 4640$, He II $\lambda 4686$, and C IV $\lambda 5808$ in both regions, testifying of the presence of late $\mathrm{WN}$ and early WC stars.

Tol 1025 - 284 — Pindao (1998) signals the presence of a broad WR bump in this galaxy.

Mrk 33 - Kunth \& Joubert (1985) list this object as having a broad He II $\lambda 4686$ excess above $0.8 \sigma$ of the background. The WR bump is confirmed by the spectra of Mas-Hesse \& Kunth $(1991,1998)$.

Mrk 178 - The SE knot of this galaxy shows broad He II $\lambda 4686$ according to González-Riestra et al. (1984). He II emission was already noted by Sargent (1972). Guseva et al. (1998) signal the presence of nebular and broad He II, and C IV $\lambda 5808$.

Mrk 1434 - Broad and nebular He II $\lambda 4686$ has been found by Izotov et al. (1997a). They also indicate the presence of low intensity broad components of $\mathrm{H} \alpha$ and/or [O III $\lambda 5007$. The reanalysis of Guseva et al. (1998) also shows C IV $\lambda 5808$ emission.

Mrk 1259 - Ohyama et al. (1997) detect broad N III $\lambda 4640$ and He II $\lambda 4686$ lines in the nuclear spectrum of this nearby starburst galaxy. Their study suggests the existence of a superwind seen nearly pole-on (see also Ohyama \& Taniguchi 1998). Guseva et al. (1998) signal also the presence of C IV $\lambda 5808$ and no nebular He II. A broad feature identified as N III $\lambda 4510$ is also indicated.

Mrk 724 - Kunth \& Schild (1984) find broad He II $\lambda 4686$ and a broad feature close to $\mathrm{C}$ IV $\lambda 5808$, as well as additional nebular lines "contaminating" the WR bump ([Fe III] $\lambda 4658$, He II $\lambda 4686$, [Ar IV] $\lambda 4711)$. The identification of C IV $\lambda 5808$ is not well established (see Kunth \& Schild 1984; Conti 1991).

NGC 3353 - Steel et al. (1996) report the presence of a broad WR bump and a possible detection of C IV $\lambda 5808$ in region $A$. These signatures are confirmed by Huang et al. (1998) who also find indications for nebular He II $\lambda 4686$.

NGC 3367 - The presence of a broad WR bump in the LINER nucleus has been signaled by Ho et al. (1995), who also note unusually broad and asymmetric emission lines (e.g. $\mathrm{H} \beta)$.

NGC 3395 - Weistrop et al. (1998) signal the presence of broad He II $\lambda 4686$ and C IV $\lambda 5808$. 
NGC 3396 - Weistrop et al. (1998) signal the presence of broad He II $\lambda 4686$ and $\mathrm{C}$ IV $\lambda 5808$, as well as nebular He II $\lambda 4686$.

Mrk 1271 - The spectrum of Izotov \& Thuan (1998) shows weak nebular and broad He II $\lambda 4686$ features. Although very weak, C IV $\lambda 5808$ could also be present according to Guseva et al. (1998). Contini (1996) only finds nebular emission lines.

SBS 1054+365 - Broad He II $\lambda 4686$ has been detected by Izotov et al. (1997a). This is confirmed by the reanalysis of Guseva et al. (1998).

Mrk 36 - The spectrum of Izotov \& Thuan (1998) shows nebular and broad He II $\lambda 4686$ features. No WR signature was detected by Campbell et al. (1986) and MasHesse \& Kunth $(1991,1998)$.

NGC 3690 - Ho et al. (1995) signaled the presence of WR features in several regions of this galaxy (=Arp 299); they exclude WR features in the nucleus. Vacca (1996, private communication) signals the presence of broad He II $\lambda 4686$ in Arp 299B and Arp 299C which are part of this complex system.

NGC 3738 - Martin (1997) points out the presence of broad He II $\lambda 4686$.

UM 439 - Automatic detection by Masegosa et al. (1991). Also listed as WR candidate by Pindao et al. (1999).

Mrk 182 - Broad He II $\lambda 4686$ is pointed out by Guseva et al. (1998). The spectrum may be too noisy to establish the presence/absence of a nebular component.

Mrk 1450 - Nebular and broad He II $\lambda 4686$, and C IV $\lambda 5808$ have been found by Izotov et al. (1994) (cf. also Guseva et al. 1998).

Mrk 1304 - Automatic detection of He II by Masegosa et al. (1991), later confirmed by Pindao (1998). Nebular and broad He II $\lambda 4686$, and C IV $\lambda 5808$ have been found by Guseva et al. (1998). The S/N appears fairly low for the latter assertion.

Mrk 1305 - Broad He II $\lambda 4686$ and C IV $\lambda 5808$ have been found by Guseva et al. (1998). The S/N appears fairly low for the latter assertion.

Mrk 750 - He II $\lambda 4686$ emission was first signaled by Kunth \& Joubert (1985). According to Conti (1991) N III $\lambda 4640$ is also detected in a spectrum of Salzer. These detections are consistent with the spectrum of Izotov \& Thuan (1998) showing a broad WR bump and nebular He II. The reanalysis of Guseva et al. (1998) also reveals C IV $\lambda 5808$ emission.

Pox 4 - Kunth \& Joubert (1985) list this object as having a broad He II $\lambda 4686$ excess above $0.8 \sigma$ of the background. This object is also included in the studies of Campbell et al. (1986) and Masegosa et al. (1991) ${ }^{3}$, which signal a possible WR feature in one or two regions.

\footnotetext{
${ }^{3}$ Campbell et al. refer to C 1148 - 203. In Masegosa et al. the name C 1148-2020 (see their Table 1) and erroneously Tol $1148-202$ (Table 2) is used. The proper identification of this
}

The reanalysis of Pindao (1998) confirms the WR bump in one region. Broad He II $\lambda 4686$ has been measured by Vacca \& Conti (1992) in two regions. The existence of a broad component is not well established (e.g. Kunth \& Sargent 1981). Nebular He II $\lambda 4686$ is most likely present in region $A$ (see spectrum of Vacca \& Conti 1992). A broad asymmetric emission component of [O III $\lambda 25007$ has been found by Méndez \& Esteban (1997).

UM 461 - Conti (1991) reports the presence of broad He II $\lambda 4686$ and relatively strong nebular [Ar IV] lines in a spectrum from Salzer.

Mrk 1307 - Izotov \& Thuan (1998) indicate the presence of broad He II $\lambda 4686$ and nebular He II (cf. Guseva et al. 1998). The presence of a broad component appears somewhat marginal (see also Contini 1996).

Mrk 193 - The analysis of Guseva et al. (1998) indicates the presence of broad and nebular He II $\lambda 4686$ in this object; the broad component was not signaled by Izotov et al. (1994).

ISZ 59 - Kunth \& Joubert (1985) list this object as having a broad He II $\lambda 4686$ excess above $0.8 \sigma$ of the background.

NGC 3995 - Weistrop et al. (1998) signal the presence of broad He II $\lambda 4686$ and C IV $\lambda 5808$, as well as nebular He II $\lambda 4686$.

NGC 4038 - From ten giant HiI regions surveyed by Rosa \& D'Odorico (1986) in this galaxy of the Antennae, one exhibits a broad emission feature at the blue WR bump.

SBS $1211+540$ - The reanalysis of Guseva et al. (1998) shows nebular and broad He II $\lambda 4686$; Izotov et al. (1994) only signaled nebular emission.

NGC 4214 - WR signatures in this galaxy were found independently by Mas-Hesse \& Kunth (1991; cf. also 1998) and Sargent \& Fillippenko (1991). The observations of the former show a broad WR bump around $4650 \AA$ and $\mathrm{C}$ IV $\lambda 5808$ due to WC stars. The latter detect WR signatures of N III $\lambda 4640, \mathrm{C}$ III $\lambda 4650$, possibly also C III $\lambda 4658$, and He II $\lambda 4686$ in two knots. From the $\mathrm{C}$ lines they also suspected the presence of WC stars. Broad He II $\lambda 4686$ and $\mathrm{C}_{\mathrm{IV}} \lambda 5808$ in several regions has also been signaled by Martin \& Kennicutt (1997). A broad H $\alpha$ component, attributed to WN stars, was also detected by Sargent \& Fillippenko in one knot. Recent UV spectroscopy of NGC 4214 with HST was obtained by Leitherer et al. (1996). Detailed spectroscopic spatial mapping by MaízApellániz et al. (1998) shows the presence of He II $\lambda 4686$ (broad and narrow) in several regions of NGC 4214.

NGC 4216 - From five giant HiI regions surveyed by Rosa \& D'Odorico (1986) in this galaxy two exhibit a broad emission feature at the blue WR bump.

Cambridge object (see Telles et al. 1997) is IRAS $11485-2018$ $=$ Pox 4 according to NED. 
NGC 4236 - González-Delgado \& Perez (1994) report the presence of broad N III $\lambda 4640$ and He II $\lambda 4686$ features in their HII region III.

M 106 - Castellanos et al. (1998) point out the presence of broad He II $\lambda 4686$ in the brightest HiI region of this Seyfert 2 galaxy.

SBS 1222+614 - Nebular and broad He II $\lambda 4686$, and C IV $\lambda 5808$ have been found by Izotov et al. (1997a) (cf. Guseva et al. 1998).

NGC 4385 - WR signatures of N III $\lambda 4640$, He II $\lambda 4686$ and possibly also C IV $\lambda 5808$ have been detected by Campbell \& Smith (1986). The first two lines are also found in the spectrum of Durret \& Tarrab (1988), who signal also a possible detection of $\mathrm{C}$ III $\lambda 4658$. The findings are confirmed by Conti (1991) according to a spectrum of Salzer; according to Conti C III $\lambda 4658$ has more likely to be identified with [Fe III] $\lambda 4658$.

II Zw 62 - Kunth \& Joubert (1985) list this object as having a broad He II $\lambda 4686$ excess above $0.8 \sigma$ of the background.

Mrk 209 - Nebular and broad He II $\lambda 4686$ have been found by Izotov et al. (1997a); the reanalysis of Guseva et al. (1998) also reveals C IV $\lambda 5808$ emission, although very weak.

NGC 4449 - Martin \& Kennicutt (1997) indicate the presence of broad He II $\lambda 4686$ and C IV $\lambda 5808$ in several regions of this object.

NGC 4532 - The presence of a weak broad WR bump has been signaled by Ho et al. (1995), who also note a very weak broad $\mathrm{H} \alpha$ component.

Mrk 1329 - Broad He II $\lambda 4686$ and a possible detection of C IV $\lambda 5808$ are signaled by Guseva et al. (1998). He II $\lambda 5412$ emission is also pointed out.

Tol 1235 - 350 — Pindao (1998) signals the presence of a broad WR bump in this galaxy.

NGC 4670 - Mas-Hesse \& Kunth (1991, 1998) report the presence of a WR bump in this galaxy; the latter publication provides only an upper limit.

Tol 1247 - 232 - Automatic detection of broad He II $\lambda 4686$ by Masegosa et al. (1991) confirmed by the analysis of Pindao (1998).

SBS 1249+493 - The reanalysis of Guseva et al. (1998) indicates the presence of nebular and broad He II $\lambda 4686$; previously only a narrow component was detected (Thuan et al. 1995).

NGC 4861 - The spectrum of Dinerstein \& Shields (1986) shows a broad WR feature centered at $4686 \AA$ and a possible detection of C IV $\lambda 5808$ (but cf. Conti 1991). The WR bump is confirmed by Mas-Hesse \& Kunth (1991, 1998), Motch et al. (1994) who also find nebular He II, and by Martin \& Kennicutt (1997) who possibly also find C IV $\lambda 5808$. The spectrum of Izotov et al. (1997a) shows broad and nebular He II, as well as C IV $\lambda 5808$ (cf. Guseva et al. 1998).

Tol 30 - Broad N III $\lambda 4640$, He II $\lambda 4686$, and probably also nebular He II have been found by Contini (1996). The exact region is not specified. Pindao et al. (1999) signal the possible presence of the WR bump in the HII region Tol 1303-281 NW associated with this galaxy.

Pox 120 - Kunth \& Joubert (1985) list this object as having a broad He II $\lambda 4686$ excess above $0.8 \sigma$ of the background.

Pox 139 - Kunth \& Joubert (1985) list this object as having a broad He II $\lambda 4686$ excess above $0.8 \sigma$ of the background. This finding is confirmed by the observations of Vacca \& Conti (1992).

NGC 5068 - From five giant Hit regions surveyed by D'Odorico et al. (1983) in this galaxy, two exhibit a broad emission feature at the blue WR bump.

SBS 1319+579 — Nebular and broad He II $\lambda 4686$ have been found by Izotov et al. (1997a) In their reanalysis Guseva et al. (1998) also detect C IV $\lambda 5808$.

NGC 5128 - The observations of six Hir regions in the elliptical galaxy Cen A (classified as Seyfert 2 in NED) by Möllenhoff (1981) revealed several WR features in one region (\# 13) near the rim of the dust disk of this well studied galaxy. Broad lines of N III $\lambda 4640$, He II $\lambda 4686$, C III $\lambda 4650$, and C IV $\lambda 5808$ are identified. According to Rosa \& D'Odorico (1986), WR features are found in two (including \# 13) out of six surveyed regions.

Pox 186 - Kunth \& Joubert (1985) list this object as having a broad He II $\lambda 4686$ excess above $0.8 \sigma$ of the background.

Tol 35 - Kunth \& Joubert (1985) list this object as having a broad HeII $\lambda 4686$ excess above $0.8 \sigma$ of the background. Campbell et al. (1986) indicate only nebular He II. The WR feature is confirmed by the spectrum of Campbell \& Smith (1986), by Masegosa et al. (1991), Vacca \& Conti (1992), and Pindao (1998). Broad asymmetric emission components of $\mathrm{H} \alpha$ and [O III] $\lambda 5007$ have been found by Méndez \& Esteban (1997).

M 83 - From eight giant HII regions surveyed by Rosa \& D'Odorico (1986) in this galaxy, three exhibit a broad emission feature at the blue WR bump.

NGC 5253 - This is a well studied amorphous galaxy. WR features have not been detected by Rosa \& D'Odorico (1986); four regions surveyed. The first reports of a broad WR bump in this galaxy are from Campbell et al. (1986) and Walsh \& Roy (1987). The high S/N observations of Schaerer et al. (1997) reveal broad features of N III $\lambda 4640$, He II $\lambda 4686$, and C IV $\lambda 5808$ in two regions, testifying of the presence of late-type WN and early-type WC stars. Nebular He II $\lambda 4686$ is very likely present in their region $A$; a region of exceptionally strong He II $\lambda 4686$ (probably nebular) was also found (see Schaerer et al. 1999). The WR bump was also detected by Kobulnicky et al. (1997), Martin \& Kennicutt (1997), Mas-Hesse \& Kunth (1998) and Pindao (1998).

Mrk 67 - Conti (1991) reports a possible detection of a weak and broad He II $\lambda 4686$ emission feature from a spectrum of Salzer. 
Mrk 1486 - Nebular and broad He II $\lambda 4686$ have been found by Izotov et al. (1997a).

Tol 89 - Durret et al. (1985) report the detection of a broad WR bump (likely N III $\lambda 4640$ and He II $\lambda 4686$ ) in this giant HII region of NGC 5398. IUE spectra also indicate the presence of WR stars (see Durret et al. 1985; Conti 1991), possibly also of the WC type. Pindao (1998) also signals the presence of the WR bump. The high $\mathrm{S} / \mathrm{N}$ observations of Schaerer et al. (1999) reveal broad features of N III $\lambda 4640$, He II $\lambda 4686$, and exceptionally strong $\mathrm{C}$ IV $\lambda 5808$, testifying of the presence of late-type WN and early-type WC stars. These authors also suspect a contribution from nebular He II $\lambda 4686$.

NGC 5430 - Keel (1982) reports a broad WR bump (likely N III $\lambda 4640$ and He II $\lambda 4686$ ) in the spectrum of a bright HII region, SE of the nucleus of this barred galaxy.

NGC 5408 - WR features have not been detected by Rosa \& D'Odorico (1986); two regions surveyed. Masegosa et al. (1991) signal a possible detection of broad He II $\lambda 4686$ in their region $B$. According to Motch et al. (1994) the He II emission is only nebular and is mostly found in two regions (their \# 3 and 4). Kovo \& Contini (1998) report both broad and nebular He II $\lambda 4686$ in two HiI regions of this galaxy with a faint broad N III $\lambda 4640$ in one of them. The nebular lines of [Fe III] $\lambda 4658$ [Ar IV] $\lambda 4711$, and $[\mathrm{Ar}$ IV] $\lambda 4740$ are strong.

NGC $5457=$ M 101 - Rosa \& D'Odorico (1986) report of seven surveyed HII regions with five detections of WR features from the spectra of D'Odorico et al. (1983). According to the latter, however, only two regions, Hodge 40 and NGC 5461 (see below), show clear broad He II $\lambda 4686$ features; the remaining objects required confirmation with spectra at higher resolution. Although uncertain, broad He II $\lambda 4686$ emission in Hodge 40 was also pointed out by Rayo et al. (1982).

NGC 5461 - The presence of broad N III $\lambda 4640$ and He II $\lambda 4686$ in this giant HiI region of M101 was first pointed out by Rayo et al. (1982) and later confirmed by D'Odorico et al. (1983).

NGC 5471 - Rayo et al. (1982) find a weak He II $\lambda 4686$ feature in this His region of M 101. No information on its width is given. D'Odorico et al. (1983) detect unbroadened lines identified as C III $\lambda 4658$ [Fe III] $\lambda 4658$, He II $\lambda 4686, \mathrm{He} \mathrm{I}+[\mathrm{Ar} \mathrm{IV}] \lambda 4711$ and $[\mathrm{Ar} \mathrm{IV}] \lambda 4740$, whose origin is likely nebular. Mas-Hesse \& Kunth $(1991,1998)$ mention the presence of a WR bump.

SBS 1408+551A - Nebular and broad He II $\lambda 4686$, and C IV $\lambda 5808$ have been found by Izotov et al. (1996).

CGCG 219 - 066 - Nebular and broad He II $\lambda 4686$ emission is found according to Izotov et al. (1998); no broad features had been signaled by Thuan et al. (1995).

Mrk 475 - Conti (1991) reports a detection of a moderately strong He II $\lambda 4686$ emission feature and possible N III $\lambda 4640$ from a spectrum of Salzer. The similarity with the spectrum of Mrk 750 is pointed out. Nebular and broad He II $\lambda 4686$, and C IV $\lambda 5808$ have been found by Izotov et al. (1994) (cf. Guseva et al. 1998). Their spectrum also show the presence of an unidentified broad feature at $\sim 4200 \AA$.

Mrk 477 - Heckman et al. (1997) detect the presence of a broad He II $\lambda 4686$ component attributed to WR stars together with several other stellar signatures in the nucleus of this powerful Seyfert 2 galaxy.

Tol 1457 - 262A - A broad He II $\lambda 4686$ emission line has been reported by Contini (1996) in one of the members of the galaxy pair Tol $1457-262$. A broad WR bump is also detected by Pindao (1998).

Tol 1457 - 262B - Whereas Contini (1996) signals the presence of a purely nebular He II $\lambda 4686$ emission, a broad WR bump is detected by Pindao (1998).

SBS 1533+574B - Nebular and broad He II $\lambda 4686$, and also C IV $\lambda 5808$ have been found in the reanalysis of Guseva et al. (1998); no broad features had been signaled by Izotov et al. (1997a).

IC 4662 - From two giant HiI regions surveyed by Rosa \& D'Odorico (1986) in this nearby galaxy, two exhibit a broad emission feature at the blue WR bump. This is also confirmed by the study of Heydari-Malayeri et al. (1991). Richter \& Rosa (1991) detect He II $\lambda 4686$ and C IV $\lambda 5808$ in one cluster. The WR bump was also detected by Mas-Hesse \& Kunth (1998).

NGC 6500 - Broad He II $\lambda 4686$ may be tentatively detected in the LINER nucleus of this galaxy (Barth et al. 1997).

Fairall 44 - Broad He II $\lambda 4686$ C IV $\lambda 5808$ and N III $\lambda 4640$ emission lines are detected in this dwarf galaxy (Kovo \& Contini 1998).

NGC 6764 - Osterbrock \& Cohen (1982) point out the presence of broad N III $\lambda 4640$ and He II $\lambda 4686$ in the spectrum of the nucleus of this barred spiral galaxy also classified as LINER or Seyfert 2. Conti (1991) argues that the line at $4660 \AA$ in their spectrum is likely [Fe III] $\lambda 4658$. The spectrum of Eckart et al. (1996) confirms the broad features of Osterbrock \& Cohen. They also signal excessive widths of $\mathrm{He} \mathrm{I} \lambda 5876$ and $\mathrm{H} \alpha$ which they attribute to emission from WR stars. Broad C III $\lambda 5696$ and C IV $\lambda 5808$ lines from WC stars are reported in the nucleus of this galaxy by Kunth \& Contini (1998).

Tol 1924 - 416 - Pindao (1998) signals the presence of a broad WR bump in this galaxy. Kovo \& Contini (1998) indicate only nebular He II $\lambda 4686$.

IC 4870 - The presence of a broad WR bump is signalled by Joguet \& Kunth (1999) in this galaxy classified as starburst or Seyfert 2. The spectral range at $\lambda>$ $5300 \AA$ was not covered.

IC 5154 - Joguet \& Kunth (1999) note the presence of broad He II $\lambda 4686, \mathrm{~N}$ III $\lambda 4640$, and $\mathrm{C}$ IV $\lambda 5808$ in one of the two nuclei of this galaxy which they classify as starburst although previously known as Seyfert 2 .

ESO 108 - IG 01 - The presence of broad He II $\lambda 4686$ and C IV $\lambda 5808$ is signalled by Joguet \& Kunth (1999) in this galaxy classified as starburst or Seyfert 2 . 
Mrk 309 - Osterbrock \& Cohen (1982) point out the presence of broad N III $\lambda 4640$ and He II $\lambda 4686$ in this Seyfert 2 galaxy. Conti (1991) argues that the line at $4660 \AA$ is likely [Fe III] $\lambda 4658$. C IV $\lambda 5808$ and C III $\lambda 5696$ emission, attributed to WC stars, is also tentatively detected by Osterbrock \& Cohen.

Mrk 315 - Kunth \& Joubert (1985) list this object as having a broad He II $\lambda 4686$ excess above $0.8 \sigma$ of the background.

ESO 148-IG 02 - WR features (N III $\lambda 4640$, He II $\lambda 4686)$ in this infrared galaxy have been reported by Johansson \& Bergvall (1988).

III Zw 107 - Kunth \& Joubert (1985) list this object as having a broad He II $\lambda 4686$ excess above $0.8 \sigma$ of the background in the southern component.

Mrk 930 - Broad He II $\lambda 4686$ has been found by Izotov \& Thuan (1998). The reanalysis of Guseva et al. (1998) also reveals a nebular He II component and broad $\mathrm{C}$ IV $\lambda 5808$. The quality of the spectrum appears fairly low for the latter assertion.

NGC 7714 - Van Breugel et al. (1985) reported weak WR features near $4686 \AA$ and possible nebular He II emission in the nucleus of this "prototypical starburst" galaxy, and call attention to the similarity with Minkowski's object (above) and extragalactic HiI regions. New long-slit observations at several position angles were obtained by González-Delgado et al. (1995), confirming the presence of broad He II $\lambda 4686$ in the nucleus. Nebular emission can also be suspected from their spectrum. From the same observations García-Vargas et al. (1997) find broad WR bumps $(\sim 4660 \AA)$ in three extra-nuclear giant HiI regions. One of them shows a definite detection of C IV $\lambda 5808$; for the remaining regions upper limits on C IV $\lambda 5808$ are given. Pindao (1998) also signals the presence of a broad WR bump; no information about the observed location given.

\section{HII galaxies showing only nebular He II emission}

HII regions ("local" or extragalactic objects) and HII galaxies showing nebular He II $\lambda 4686$ are considered to be quite rare (e.g. Garnett et al. 1991). Although not intended as a complete inventory these authors quote only 17 objects drawn from the studies of dwarf emission-line galaxies by Kunth \& Sargent (1983) and Campbell et al. (1986). Many new observations revealing nebular He II $\lambda 4686$ emission have since been found, mostly in the sample of Izotov and collaborators (see Sect. 2).

In Table 2 we list extragalactic HII regions showing He II $\lambda 4686$ emission which is entirely attributed to nebular emission processes. In some cases, however, WR signatures are suspected by some authors; see Col. 8 for references. We restrict ourself to objects from the Terlevich et al. (1991) catalogue analysed by various authors and the sample of Izotov and collaborators, since these constitute the largest available samples of such objects. All except one object (Pox 105) from Kunth \& Sargent (1983) showing He II $\lambda 4686$ emission was later classified as a WR galaxy (Kunth \& Joubert 1985 and references in Sect. 3). From the four emission line galaxies with narrow He II $\lambda 4686$ emission listed by Conti (1991, his Table 2) three are now classified as WR galaxies (see Table 1 ); the presence of a broad He II $\lambda 4686$ component due to WR stars is suspected in the Seyfert 2 galaxy Pox 52 (Kunth et al. 1987).

From the 12 objects of Campbell et al. (1986) for which a measurement is available, six remain in this category, although two additional objects are suspected to show broad He II. In the other objects from Campbell et al., WR features have been found by other investigators (see Sect. 3), in part also in re-analysis of the same observational data (e.g. Masegosa et al. 1991).

From the sample of Izotov and collaborators (including 60 objects published up to 1998, i.e. including Izotov \& Thuan 1998), 40 show nebular He II. However only 9 objects (listed in Table 2) reveal no broad 4686 component according to these studies and the reanalysis of Guseva et al. (1998).

\section{Suspected WR galaxies and galaxies without He II $\lambda 4686$}

\subsection{Suspected WR galaxies}

Candidate WR galaxies resulting from some of the searches discussed in Sect. 2 or found loosely in the literature are listed in Table 3. The reader is referred to the original papers for the justification of the possible presence of WR stars.

Most of the suspected WR galaxies come from the spectrophotometric catalogue of His galaxies of Terlevich et al. (1991), following the analysis of Masegosa et al. (1991) or Pindao et al. (1999) which also includes new objects. As stated earlier, from Masegosa et al. (1991) we take the subset of objects included in their Table 1 but not in Table 2 as candidates. Although partly based on the same observations the analysis of Masegosa et al. (1991) and Pindao et al. (1999) do not always yield the same candidates. We have retained all objects classified as "candidate" by either one of these studies. New observations will be necessary to establish the definite presence or absence of WR features.

Few objects from Vacca \& Conti (1992) have only an upper limit on broad He II $\lambda 4686$. For most of them independent observations are now available. Otherwise these objects are retained as suspected WR galaxies.

In some studies of IUE spectra strong UV P-Cygni lines of $\mathrm{N}$ and $\mathrm{C}(\mathrm{N}$ V $\lambda 1240, \mathrm{C}$ IV $\lambda 1550)$ have been interpreted as signatures of WR stars in theses objects (e.g. Durret et al. 1985; Lamb et al. 1990). However, these lines are also strong in $\mathrm{O}$ stars and hence cannot be used as 
a clear diagnostic for WR stars (cf. Leitherer et al. 1995). Therefore, objects suspected on these grounds have not been included in Table 3 .

In rare cases, WR stars have also been suspected on indirect grounds (e.g. M100: Wozniak et al. 1998).

Interestingly, a broad WR bump is suggested to be present in the optical spectrum of two distant central cluster galaxies with strong cooling flows (Abell 1068 and 1835; Allen 1995). The recent study of Contini et al. (1998), however, casts serious doubt about the reality of a broad feature in Abell 1835. If true, these objects with redshifts $z \sim 0.14$ and 0.25 respectively, represent the most distant objects known to date where WR stars have been detected from (rest-frame) optical spectra. High redshift galaxies $(z \sim 3)$ may, however, also show WR signatures (see Sect. 7).

\subsection{Emission line galaxies without HeII}

Conti (1991, his Table 3) lists a sample of emission line galaxies which have properties similar to WR galaxies, and where a search for He II $\lambda 4686$ emission (broad or narrow) has been made with negative results. For obvious reasons such a list is necessarily incomplete and the inclusion in such a list also strongly depends on the sensitivity $(\mathrm{S} / \mathrm{N}$, resolution etc.) of the data. We therefore renounce on such a compilation. However, few updates are appropriate on some objects from Table 3 of Conti (1991).

Mrk 1087 - While Vacca \& Conti (1992) provide only an upper limit on He II $\lambda 4686$, a detection is provided in the recent spectra of Vaceli et al. (1997). No information on the width of this line is given. Retained as suspected WR galaxy.

Mrk 1094 - We have retained the criteria of Kunth \& Joubert (1985) and hence included this object in Table 1 . No new observations published.

$\mathbf{0 8 3 3}+\mathbf{6 5 2}=$ IRAS 08339+6517 - From their spectrum Veilleux et al. conclude that no WR features are present in this galaxy $(=0833+652$ in Conti 1991).

Tol $2=$ Tol $0957-278-$ Same comment as for Mrk 1094.

Tol $9=$ Tol $1032-\mathbf{2 8 3}-$ Although no broad He II $\lambda 4686$ feature seems present in this object (Kunth \& Schild 1986) we list in the category of suspected WR galaxies based on the possible detection of other broad features shortward of $5876 \AA$ (Kunth \& Schild 1986).

Other objects for which an upper limit on broad WR features is given or where a non-detection is signaled are found in publications issued from the systematic searches discussed in Sect. 2.

\section{Discussion and conclusion}

We have presented an up-to-date and presumably quite complete compilation of WR galaxies from the literature.
The number of such objects has considerably increased in the last years and now totals 139 (Table 1 ). In addition to broad He II $\lambda 4686$, the basic "classification line" for WR galaxies, we include, for the first time, relevant information about the presence of various other broad emission lines. In particular the presence of C IV $\lambda 5808$ emission originating essentially from WC stars is now detected in many objects.

A large fraction of WR galaxies also show nebular He II $\lambda 4686$ emission, indicative of high excitation. Where available we also include information about this line. Conversely, few objects are known which show only nebular He II emission, i.e. no apparent signs of broad stellar emission features. A list of these extra-galactic HiI regions is provided in Table 2. This may in particular be used to investigate the possible link between the phenomenon of high excitation and the presence of WR stars as suggested e.g. by Schaerer (1996).

We have also compiled a list of objects suspected to harbour WR stars (Table 3). This could serve for future follow-up spectroscopy.

Most of the work on WR galaxies and related objects discussed above is based on spectroscopy in the visible. Are populations of WR stars also detectable at other wavelengths?

In the infrared no direct signature of WR populations have been detected to the best of our knowledge. The most likely explanations are that 1) many strong features of WR stars coincide with strong nebular lines (e.g. He I $2.06 \mu \mathrm{m}$, $\operatorname{Br} \gamma$ ), and 2) WR features in the IR are strongly diluted by cool stars, which contribute the bulk of the emission at these wavelengths.

The strongest indicator of WR stars in UV is the presence of broad He II $\lambda 1640$ emission which is not seen in emission in other stars. Predictions of its strength and the expected line profile for integrated populations are given by Leitherer et al. (1995) and Schaerer \& Vacca (1998). The use of this line is, however, not straightforward for several reasons. Potential difficulties specific to IUE spectra have been discussed by Leitherer et al. (1995). He II $\lambda 1640$ is detected in the average spectra of $\sim 20$ starbursts (including several WR galaxies) of low and high metallicity of Heckman et al. (1998). In particular the spectra illustrate also the presence of multiple stellar and interstellar absorption lines in this wavelength range, which complicates the quantitative use of this line for diagnostics.

UV high resolution and high $\mathrm{S} / \mathrm{N}$ spectra obtained with HST indeed show the presence of this emission line in known WR galaxies (e.g. NGC 4214, NGC 1741; Conti et al. 1996; Leitherer et al. 1996). Interestingly these objects show a close resemblance with recently discovered high redshift galaxies (cf. Steidel et al. 1996; Ebbels et al. 1996; Lowenthal et al. 1997). In addition to the strongest stellar UV lines ( $\mathrm{Si}$ IV, C IV wind lines) the average spectrum of Lowenthal et al. of 11 objects with $z \sim 3$ also 
shows the He II $\lambda 1640$ emission line! Quantitative studies of stellar populations including possibly WR stars should be possible in the future using these lines.

The numerous recent findings of massive stars in Seyfert 2 and LINERs, as well as detections of high redshift galaxies exhibiting signatures of massive stars further stress the need to deepen our understanding of the physical processes in "local objects", and illustrate the interest of studies on massive stars and their interactions with the ISM, stellar populations, and starbursts in a wider context. It is the hope that our compilation will provide a useful basis for such undertakings.

Acknowledgements. We thank Rosa González-Delgado, Daniel Kunth, Cristal Martin, and Bill Vacca for useful communications and discussions, and Peter Conti, Daniel Kunth, Claus Leitherer and Yuri Izotov for comments on an earlier version of the manuscript. Yuri Izotov, Benoit Joguet, Bill Vacca, and Donna Weistrop, kindly provided results before publication. Some comments and corrections were made during the IAU Symposium 193 by several colleagues. We thank them here for their help. This research has made extensive use of the NASA Extragalactic Database (NED), the NASA Astrophysics Data System (ADS) Article Service, and SIMBAD, which is operated by the CDS in Strasbourg, France. DS acknowledges a grant from the Swiss National Foundation of Scientific Research.

\section{References}

Allen S.W., 1995, MNRAS 276, 947

Allen D.A., Wright A.E., Goss W.M., 1976, MNRAS 177, 91

Armus L., Heckman T.M., Miley G.K., 1988, ApJ 326, L45

Arnault P., Kunth D., Schild H., 1989, A\&A 224, 73

Barth A.J., Reichert G.A., Ho L.C., Shields J.C., Filippenko A.V., Puchnarewicz E.M., 1997, AJ 114, 2313

Van Breugel W., Filippenko A.V., Heckman T., Miley G., 1985, ApJ 293, 83

Breysacher J., Azzopardi M., Testor G., Muratorio G., 1997, A\&A 326, 976

Campbell A.W., Smith L.J., 1986, in "Luminous Stars and Associations in Galaxies", Loore C., Willis A.J., Laskarides P. (eds.), IAU Symp. 116. Kluwer: Netherland 499

Campbell A., Terlevich R., Melnick J., 1986, MNRAS 223, 811

Castellanos M., Diaz A.I., Terlevich E., Garcia-Vargas M.L., 1998, in "ESO conference on Chemical Evolution from Zero to High Redshift", Lecture Notes in Physics. Springer Verlag (in press)

Conti P.S., 1991, ApJ 377, 115

Conti P.S., Leitherer C., Vacca W.D., 1996, ApJ 461, L87

Conti P.S., Vacca W.D., 1994, ApJ 423, L97

Contini T., in "WR Stars in the Framework $f$ Stellar Evolution", 33rd Liège Int. Astroph. Coll., de Vreux J.M. et al. (eds.). Liège: Université de Liège, p. 619

Contini T., Davoust E., Considere S., 1995, A\&A 303, 440

Contini T., et al., 1998 (in preparation)

De Mello D., Schaerer D., Heldmann J., Leitherer C., 1998, ApJ 507, 199
Dinerstein H.L., Shields G.A., 1986, ApJ 311, 45

D’Odorico S., Rosa M., Wampler E.J., A\&AS 53, 97

Drissen L., Roy J.-R., 1994, PASP 106, 974

Drissen L., Roy J.-R., 1996, in "From Stars to Galaxies: The Impact of Stellar Physics on Galaxy Evolution", Leitherer C., Fritze-v. Alvensleben U., Huchra J. (eds.), ASP Conf. Ser. 98, 406

Drissen L., Roy J.-R., Moffat A.F.J., 1993, AJ 106, 1460

Duc P.-A., Mirabel I.F., 1998, A\&A 333, 813

Durret F., Bergeron J., Boksenberg A., 1985, A\&A 143, 347

Durret F., Tarrab I., 1988, A\&A 205, 9

Ebbels T.M.D., Le Borgne J.-F., Pello R., et al., 1996, MNRAS 281, L75

Eckart A., Cameron M., Boller T., et al., 1996, ApJ 472, 588

Eichendorf W., Nieto J.-L., 1984, A\&A 132, 342

Evans I.N., Dopita M.A., 1986, ApJ 310, L15

Garcia-Vargas M.L., Gonzalez-Delgado R.M., Perez E., et al., 1997, ApJ 478, 112

Garnett D.R., Kennicutt R.C.J., Chu Y.-H., Skillman E.D., 1991, ApJ 373, 458

Gonzalez-Delgado R.M., Perez E., 1994, in "Violent Star Formation from 30 Doradus to QSO's", Tenorio-Tagle G. (ed.). Cambridge Univ. Press, p. 289

Gonzalez-Delgado R.M., Perez E., Diaz A.I., et al., 1995, ApJ 439, 604

Gonzalez Delgado R.M., Leitherer C., Heckman T., Cervino M., 1997, ApJ 483, 705

Gonzalez-Delgado R.M., Perez E., Tenorio-Tagle G., et al., 1994, ApJ 437, 239

González-Riestra R., Rego M., Zamorano J., 1984, A\&A 202, 27

Guseva N.G., Izotov Y.I., Thuan T.X., 1998 (in preparation)

Heckman T.M., Gonzalez-Delgado R., Leitherer C., Meurer G.R., Krolik J., Wilson A.S., Koratkar A., Kinney A., 1997, ApJ 482, 114

Heckman T.M., Robert C., Leitherer C., Garnett D.R., Van Der Rydt F., 1998, ApJ 503, 646

Heydari-Malayeri M., Melnick J., Martin J.-M., 1990, A\&A 234, 99

Hickson P., 1982, ApJ 255, 382

Ho L.C., Filippenko A.V., Sargent W.L., 1995, ApJS 98, 477

Huang J.H., Gu Q.S., Ji L., Wei J.Y., Zheng W., 1998, ApJ (submitted)

Huang J.H., Gu Q.S., Su H., Shang Z., 1996, Ap\&SS 235, 109 van der Hucht K.A., 1996, in "WR Stars in the Framework $f$ Stellar Evolution", 33rd Liège Int. Astroph. Coll., de Vreux J.M. et al. (eds.). Liège: Université de Liège, p. 17

van der Hucht K.A., et al., 1998 (in preparation)

Hunter D.A., Thronson H.A.J., 1995, ApJ 452, 238

Hutsemekers D., Surdej J., 1984, A\&A 133, 209

Izotov Y.I., Dyak A.B., Chaffee F.H., Foltz C.B., Kniazev A.Y., Lipovetsky V.A., 1996, ApJ 458, 524

Izotov Y.I., Foltz C.B., Green R.F., Guseva N.G., Thuan T.X., 1997b, ApJ 487, L37

Izotov Y.I., Lipovetsky V.A., Chaffee F.H., et al., 1997c, ApJ 476,698

Izotov Y.I., Thuan T.X., 1998, ApJ 500, 188

Izotov Y.I., Thuan T.X., Lipovetsky V.A., 1997a, ApJS 108, 1

Izotov Y.I., Thuan T.X., Lipovetsky V.A., 1994, ApJ 435, 647

Izotov Y.I., Chaffee F.H., Foltz C.B., Green R.B., Guseva N.G., 1998 (in preparation) 
Joguet B., Kunth D., 1999 (in preparation)

Johansson L., Bergvall N., 1988, A\&A 192, 81

Keel W.C., 1982, PASP 94, 765

Kobulnicky H.A., Skillman E.D., Roy J.-R., Walsh J.R., Rosa M.R., 1997, ApJ 477, 679

Kovo O., Contini T., 1998, in "Wolf-Rayet Phenomena in Massive Stars and Starburst Galaxies", van der Hucht K.A., Koenigsberger G., Eenens P.R.J. (eds.), IAU Symp. 193, San Francisco: ASP (in press)

Krueger H., Fritze-Von Alvensleben U., Fricke K.J., Loose H.-H., 1992, A\&A 259, L73

Kunth D., Contini T., 1998, in "Wolf-Rayet Phenomena in Massive Stars and Starburst Galaxies", van der Hucht K.A., Koenigsberger G., Eenens P.R.J. (eds.), IAU Symp. 193, San Francisco: ASP (in press)

Kunth D., Joubert M., 1985, A\&A 142, 411

Kunth D., Sargent W.L.W., 1981, A\&A 101, L5

Kunth D., Sargent W.L.W., 1983, ApJ 273, 81

Kunth D., Sargent W.L.W., Bothun G.D., 1987, AJ 93, 29

Kunth D., Schild H., 1986, A\&A 169, 71

Lamb S.A., Bushouse H.A., Towns J.W., 1990, in "ESA, Evolution in Astrophysics: IUE Astronomy in the Era of New Space Missions", p. 591

Legrand F., Kunth D., Roy J.-R., Mas-Hesse J.M., Walsh J.R., 1997, A\&A 326, L17

Leitherer C., Robert C., Heckman T.M., 1995, ApJS 99, 173

Leitherer C., Vacca W.D., Conti P.S., Filippenko A.V., Robert C., Sargent W.L.W., 1996, ApJ 465, 717

Maeder A., Conti P.S., 1994, ARAA 32, 227

Maiz-Apellaniz J., Mas-Hesse J.M., Munoz-Tunon C., Vilchez J.M., Castaneda H.O., 1998, A\&A 329, 409

Margon B., Anderson S.F., Mateo M., Fich M., Massey P., 1988, ApJ 334, 597

Martin C.L., 1997, ApJ 491, 561

Martin C.L., Kennicutt R.C. Jr., 1997, ApJ 483, 698

Masegosa J., Moles M., Del Olmo A., 1991, A\&A 244, 273

Mas-Hesse J.M., Kunth D., 1991, in "Wolf-Rayet Stars and Interrelations with Other Massive Stars in Galaxies", van der Hucht K.A., Hidayat B. (eds.), IAU Symp. 142. Kluwer: Netherland, p. 613

Mas-Hesse J.M., Kunth D., 1998, A\&A (in press)

Massey P., 1996, in "WR Stars in the Framework f Stellar Evolution", 33rd Liège Int. Astroph. Coll., de Vreux J.M. et al. (eds.). Liège: Université de Liège, p. 361

Meurer G.R., Heckman T.M., Leitherer C., Kinney A., Robert C., Garnett D.R., 1995, AJ 110, 2665

Meynet G., 1995, A\&A 298, 767

Möllenhoff C., 1981, A\&A 99, 341

Motch C., Pakull M.W., Pietsch W., 1994, in "Violent Star Formation from 30 Doradus to QSO's", Tenorio-Tagle G. (ed.). Cambridge Univ. Press, p. 208

Ohyama Y., Taniguchi Y., 1998, ApJ 498, L27

Ohyama Y., Taniguchi Y., Terlevich R., 1997, ApJ 480, L9

Osterbrock D.E., Cohen R.D., 1982, ApJ 261, 64

Phillips A.C., Conti P.S., 1992, ApJ 395, L91

Pindao M., 1998, in "Wolf-Rayet Phenomena in Massive Stars and Starburst Galaxies", van der Hucht K.A., Koenigsberger G., Eenens P.R.J. (eds.), IAU Symp. 193, San Francisco: ASP (in press)
Pindao M., et al., 1999 (in preparation)

Rayo J.F., Peimbert M., Torres-Peimbert S., 1982, ApJ 255, 1 Richter O.-G., Rosa M.R., 1991, in "Wolf-Rayet Stars and Interrelations with Other Massive Stars in Galaxies", van der Hucht K.A., Hidayat B. (eds.), IAU Symp. 142. Kluwer: Netherland, p. 653

Robledo-Rella V., Conti P.S., 1993, in "Massive Stars: Their Lives in the Interstellar Medium", Cassinelli J.P., Churchwell E.B. (eds.), ASP Conf. Ser. 35, 534

Rosa M., D'Odorico S., 1986, in "Luminous Stars and Associations in Galaxies", Loore C., Willis A.J., Laskarides P. (eds.), IAU Symp. 116. Kluwer: Netherland, p. 355

Rubin V.C., Ford W.K.J., Hunter D.A., 1990, ApJ 365, 86

Roy J.-R., Aube M., McCall M.L., Dufour R.J., 1992, ApJ 386, 498

Sargent W.L.W., 1972, ApJ 173, 7

Sargent W.L.W., Filippenko A.V., 1991, AJ 102, 107

Schaerer D., 1996, ApJ 467, L17

Schaerer D., 1997, in "Dwarf Galaxies: Probes for Galaxy Formation and Evolution", Andersen J. (ed.), Highlights in Astronomy (in press)

Schaerer D., Contini T., Kunth D., Meynet G., 1997, ApJ 481, $\mathrm{L} 75$

Schaerer D., Contini T., Kunth D., 1999, A\&A 341, 399

Schaerer D., de Mello D., Leitherer C., Heldmann J., 1998, in "Dwarf Galaxies and Cosmology", Thuan T.X., Balkowski C., Cayette V., Tran Than Van J. (eds.). Éditions Frontières, Gif-sur-Yvette, France (in press) (astro-ph/9805352)

Schaerer D., Vacca W.D., 1998, ApJ 497, 618

Schmidt-Kaler Th., Feitzinger J.V., 1984, in "Observational Tests of the Stellar Evolution Theory", Maeder A., Renzini A. (eds.). IAU Symp. 105, 325

Stasińska G., 1998, in "Wolf-Rayet Phenomena in Massive Stars and Starburst Galaxies", van der Hucht K.A., Koenigsberger G., Eenens P.R.J. (eds.), IAU Symp. 193, San Francisco: ASP (in press)

Steel S.J., Smith N., Metcalfe L., Rabbette M., McBreen B., 1996, A\&A 311, 721

Steidel C.C., Giavalisco M., Pettini M., Dickinson M., Adelberger K.L., 1996, ApJ 462, L17

Storchi-Bergmann T., Fernandes R.C., Schmitt H.R., 1998, ApJ 501, 94

Telles E., Melnick J., Terlevich R., 1997, MNRAS 288, 78

Terlevich R., Melnick J., Masegosa J., Moles M., Copetti M.V.F., 1991, A\&AS 91, 285

Thuan T.X., Izotov Y.I., Lipovetsky V.A., 1995, ApJ 445, 108

Thuan T.X., Izotov Y.I., Lipovetsky V.A., 1996, ApJ 463, 120

Vacca W.D., 1996 (private communication)

Vacca W.D., Conti P.S., 1992, ApJ 401, 543

Vaceli M.S., Viegas S.M., Gruenwald R., De Souza R.E., 1997, AJ 114,1345

Veilleux S., Kim D.-C., Sanders D.B., Mazzarella J.M., Soifer B.T., 1995, ApJS 98, 171

Walsh J.R., Roy J.-R., 1987, ApJ 319, L57

Walsh J.R., Roy J.-R., 1997, MNRAS 288, 726

Weistrop D., et al., 1998 (in preparation)

Wozniak H., Friedli D., Martinet L., Pfenniger D., 1998, A\&A $330, \mathrm{~L} 5$ 